\title{
Network instability dynamics drive a transient bursting period in the developing hippocampus in vivo
}

\author{
Jürgen Graf ${ }^{1,5}$, Vahid Rahmati ${ }^{1,2,3,5}$, Myrtill Majoros ${ }^{1}$, Otto W. Witte ${ }^{1}$, Christian Geis ${ }^{1,2}$, \\ Stefan J. Kiebel ${ }^{3}$, Knut Holthoff ${ }^{1,6}$ and Knut Kirmse ${ }^{1,4,6 *}$
}

1

2

3

4

$$
\text { Würzburg, Germany }
$$

5 6

These authors contributed equally.

Senior authors

Number of Figures -7

Number of Supplemental Figures -1

Number of Supplemental Tables -5

* Correspondence to:

Dr. Knut Kirmse

Department of Neurophysiology, Institute of Physiology, University of Würzburg, 97070

Würzburg, Germany

Phone: +49 (0)931 3187473

Fax: +49 (0)931 3182741

E-mail: knut.kirmse@uni-wuerzburg.de 


\begin{abstract}
Spontaneous correlated activity is a universal hallmark of immature neural circuits. However, the cellular dynamics and intrinsic mechanisms underlying neuronal synchrony in the intact developing brain are largely unknown. Here, we use two-photon $\mathrm{Ca}^{2+}$ imaging to comprehensively map the developmental trajectories of spontaneous network activity in hippocampal area CA1 in vivo. We unexpectedly find that synchronized activity peaks after the developmental emergence of effective synaptic inhibition in the second postnatal week. We demonstrate that the enhanced network synchrony reflects an increased functional coupling of individual neurons to local population activity. However, pairwise neuronal correlations are low, and network bursts recruit CA1 pyramidal cells in a virtually random manner. Using a dynamic systems modeling approach, we reconcile these experimental findings and identify network bi-stability as a potential regime underlying network burstiness at this age. Our analyses reveal an important role of synaptic input characteristics and network instability dynamics for the emergence of neuronal synchrony. Collectively, our data suggest a mechanism, whereby developing CA1 performs extensive input-discrimination learning prior to the onset of environmental exploration.
\end{abstract}

Keywords: CA1, development, two-photon $\mathrm{Ca}^{2+}$ imaging, in vivo, network modeling, stability 


\section{Introduction}

Developing neural circuits generate correlated spontaneous activity in which synchronous activations of large groups of neurons are interspersed by relatively long periods of quiescence (Molnar et al., 2020). In rodents, synchronized network activity commences long before the onset of hearing, vision and active environmental exploration and makes important contributions to the proper assembly of brain circuits (Kirkby et al., 2013). Activity-dependent refinements operate at multiple steps of maturation, including the control of neural progenitor progression (Vitali et al., 2018), apoptotic cell death (Blanquie et al., 2017; Wong et al., 2018), neuronal cell-type specification (Sun et al., 2018), migration (Maset et al., 2021) as well as synapse formation and plasticity (Oh et al., 2016; Sando et al., 2017; Winnubst et al., 2015). Experimental and theoretical evidence suggests that, in addition to the overall level of activity, specific spatiotemporal firing patterns are critical for activity-dependent refinements to occur (Zhang et al., 2011; Albert et al., 2008).

A representative example of correlated spontaneous network activity is found in the neonatal hippocampus in vivo. During the first postnatal week, the main electrophysiological signature are bursts of multi-unit activity (MUA) (Leinekugel et al., 2002), which bilaterally synchronize large parts of the dorsal CA1 and are often accompanied by sharp waves (SPWs) in the local field potential (Valeeva et al., 2020; Valeeva et al., 2019). SPWs frequently follow myotonic limb or whisker twitches (Karlsson et al., 2006; Valeeva et al., 2019; Del Rio-Bermudez et al., 2020), suggesting that SPWs convey feedback information from the somatosensory periphery. By the second postnatal week, discontinuous activity in the olfactory bulb drives network oscillations in the entorhinal cortex (Gretenkord et al., 2019), further pointing to a role of multi-sensory integration in limbic ontogenesis. Interestingly, recent in vivo investigations revealed that GABAergic interneurons (INs) could promote MUA bursts in the neonatal CA1 
through NKCC1-dependent excitation of pyramidal cells (PCs), although inhibitory effects of GABAergic signaling coexist (Graf et al., 2021; Murata et al., 2020; but see Valeeva et al., 2016). A qualitatively similar situation applies to the immature hippocampus in vitro (Ben-Ari et al., 1989; Flossmann et al., 2019), in which correlated spiking of PCs is facilitated by increasing the intracellular chloride concentration (Zhang et al., 2019; Spoljaric et al., 2019), whereas inhibition of chloride uptake has the opposite effect (Dzhala et al., 2005). In this line, in vitro studies suggest that correlated spontaneous activity largely disappears by the beginning of the second postnatal week, when the reversal potential of $\mathrm{GABA}_{A}$ receptormediated currents shifts into the hyperpolarizing direction (Tyzio et al., 2008; Spoljaric et al., 2017). However, the developmental trajectories of cellular network firing dynamics in the hippocampus in vivo remain unknown.

Using two-photon $\mathrm{Ca}^{2+}$ imaging, we here provide the first detailed analysis of the spatiotemporal dynamics of network activity in the developing CA1 region at single-cellular resolution in vivo. We reveal that CA1 PCs undergo a transient period of enhanced burst-like network activity during the second postnatal week, when GABA already acts as an inhibitory transmitter. Our results show that, at this time, network bursts (NBs) recruit CA1 PCs in an almost random manner, and recurring cellular activation patterns become more stable only after eye opening. Using computational network modeling, we identify bi-stability as a dynamical regime underpinning the enhanced bursting activity of CA1 PCs. We show that NBs mainly reflect the network's intrinsic instability dynamics, which exquisitely depend on proper input timing and strength. In addition, inhibitory GABAergic signaling effectively promotes state transitions underlying NB generation. Our data suggest a mechanism, whereby CA1 undergoes extensive input-discrimination learning before the onset of environmental exploration. 


\section{Results}

Reliable detection of somatic $\mathrm{Ca}^{2+}$ transients in densely labeled tissue

We used in vivo two-photon laser-scanning microscopy (2PLSM) in spontaneously breathing, head-fixed $E m \times 1^{I R E S c r e}: G C a M P 6 s^{L S L}$ mice to record somatic $\mathrm{Ca}^{2+}$ transients (CaTs) from CA1 PCs as a proxy of their firing activities. In this strain, Cre is expressed in virtually all CA1 PCs (Kummer et al., 2012; Gorski et al., 2002). Due to the finite point-spread function inherent to 2PLSM, dense cell labeling resulted in a non-negligible overlap of signals originating from neighboring somata and/or neurites (Denis et al., 2020; Chen et al., 2020). Our preliminary analysis revealed that, under such conditions, standard CaT detection methods based on analyzing mean fluorescence intensities from regions of interests (ROIs) can lead to substantial false positive rates (Fig. 1). We therefore devised a novel cell-specific spatial template-matching approach for the reliable detection of CaTs in densely labeled tissue, which we refer to as CATHARSiS (Calcium transient detection harnessing spatial similarity). CATHARSiS makes use of the fact that, for each cell, the spike-induced changes in GCaMP fluorescence intensity $(\Delta \mathrm{F})$ have a specific, spatially inhomogeneous (ring-like) configuration (see Methods for details). In brief, a cell-specific spatial $\Delta \mathrm{F}$ template representing the active cell is computed (Figs. $1 \mathrm{~A}$ and $1 \mathrm{~B}$ ) and optimally scaled to fit its $\Delta \mathrm{F}$ in each recorded frame. Based on the optimum scaling factor and the quality of the fit, a detection criterion $D(t)$ is computed for each time point (Clements et al., 1997). $D(t)$ is then subjected to a generalpurpose event detection routine for the extraction of CaT onsets (Rahmati et al., 2018). We first illustrate CATHARSiS by analyzing simulated spike-induced CaTs in ring-shaped cells (Figs. 1A and 1B). Here, fluorescence signals of the cell of interest were contaminated by (I) signals originating from a partially overlapping second cell, (II) spatially homogenous fluorescence changes mimicking axon-based neuropil activity (Kerr et al., 2005) and (III) a low 
level of Poissonian noise (Fig. 1C). Figures $1 \mathrm{C}$ and $1 \mathrm{D}$ demonstrate that $D(t)$ will increase only if $\Delta \mathrm{F}$ has a spatial configuration similar to that of the template, i.e. if the simulated cell is active. Of note, $D(t)$ is insensitive to a spatially uniform offset of $\Delta \mathrm{F}$ and can decrease for mean $\Delta \mathrm{F}$ increases having a dissimilar spatial configuration (\#3 in Figs. $1 \mathrm{C}$ and $1 \mathrm{D}$ ). We applied CATHARSiS to two simulated sample cells of identical shape and varied their spatial overlap from 0 to $75 \%$ of the cell area, in accordance to the observed overlap in our empirical data. CATHARSiS correctly retrieved all ground-truth CaTs without false positive events $(n=$ 665 CaTs in total). We also found that the delay of detected CaT onsets vs. simulated spikes was low (-0.3 \pm 0.0 frames), pointing to a high temporal accuracy of spike reconstruction, which is a prerequisite of a precise analysis of network activity patterns and cellular synchronicity. We next evaluated CATHARSiS on data recorded from developing CA1 PCs in Emx1 $1^{\text {IREScre }}: G C a M P 6 s^{L S L}$ mice in vivo (Figs. $1 \mathrm{E}$ and $1 \mathrm{~F}$ ). For comparison, a consensus visual annotation by human experts was used (Fig. 1G, top), as simultaneous electrophysiological data were not available (see Methods). We compared CATHARSiS to an event detection routine based on analyzing mean $\Delta \mathrm{F}(\mathrm{t})$ and found that recall was $\sim 95 \%$ for both approaches (Fig. 1I; see Table S1). However, CATHARSiS yielded considerably fewer false positive events, thus resulting in a significantly higher precision and F1 score (Fig. 1I). Importantly, the delay of detected CaT onsets relative to the consensus annotation was consistently low $(0.9 \pm$ 0.1 frames at a frame rate of $11.6 \mathrm{~Hz}, \mathrm{n}=20$ cells), confirming that CATHARSiS achieved a high temporal accuracy.

We conclude that CATHARSiS is suited for the reliable reconstruction of somatic CaTs in densely packed neuronal tissue with both high detection and temporal accuracies.

\section{A transient period of firing equalization during CA1 development in vivo}


In the adult CA1, firing rate distributions are approximately log-normal, implying that a minority of neurons accounts for the majority of spikes. In addition, firing rates of individual cells are relatively stable across brain states and tasks, suggesting that skewed firing rate distributions reflect an inherent characteristic of mature hippocampal computations (Mizuseki et al., 2013). To reveal the developmental trajectory of single-cell firing characteristics, we applied CATHARSiS to extract spontaneous CaTs from Emx1+ PCs at P3-4 (n = 19 fields of view [FOVs]), P10-12 ( $\mathrm{n}=11$ FOVs) and P17-19 ( $\mathrm{n}=12$ FOVs), respectively (Fig. 2A). For the sake of brevity, these age groups are hereafter referred to as P4, P11 and P18, respectively. We found that mean CaT frequencies significantly increased $\sim 2.5$-fold from $1.5 \pm 0.2 \mathrm{~min}^{-1}$ at $\mathrm{P} 4$ to $3.9 \pm 0.4 \mathrm{~min}^{-1}$ at $\mathrm{P} 11$ and remained relatively stable afterwards $\left(\mathrm{P} 18: 4.8 \pm 0.3 \mathrm{~min}^{-1}\right.$, Figs. 2B and 2C; see Table S2). Additionally, we observed a striking change in the shape of CaT frequency distributions, which were broad and strongly right-tailed at P4 and P18, but much less so in the second postnatal week (Fig. 2B). To quantify the dispersion of firing rates among individual cells, we plotted the corresponding Lorenz curves (Fig. 2D), in which the cumulative proportion of CaT frequencies is plotted against the cumulative proportion of cells rank-ordered by frequency (Mizuseki et al., 2013). Here, the line of equality represents the case where all neurons have equal firing rates. We computed the Gini coefficient as a measure of deviation from equality (for a graphical representation, see inset in Fig. 2D). Gini coefficients underwent a transient minimum at P11, indicating that CaT frequencies among individual neurons were considerably more similar to each other as compared to P4 and P18 (Fig. 2E). We next addressed whether these developmental alterations in firing rates were accompanied by changes in the irregularity of firing in individual cells. The local coefficient of variation (CV2), a robust measure of local spiking irregularity (Holt et al., 1996; Ponce-Alvarez et al., 2010), gradually declined from P4 to P18 (Fig. 2G). At P11, CV2 was close to one, 
indicating that the irregularity of CaT occurrence is similar to that of a Poissonian point process, in which successive events occur independently of one another. As previously observed for CaT frequencies, CV2 distributions were also relatively broad at P4 and P18, but narrow at P11 (Fig. 2F). Consistently, Gini coefficients of CV2 showed a distinct minimum at P11 (see \#4 in Table S2).

Collectively, our data reveal a transient equalization of the firing statistics of individual CA1 PCs during the second postnatal week, while highly skewed firing-rate distributions eventually emerge only around/after eye opening.

\section{CA1 undergoes a transient enhanced bursting period in vivo}

Previous in vitro work has identified giant depolarizing potentials (GDPs) as the most prominent pattern of synchronized network activity in the neonatal hippocampus (Ben-Ari et al., 1989; Leinekugel et al., 1997; Garaschuk et al., 1998). GDPs depend on a depolarizing action of $\mathrm{GABA}_{\mathrm{A}}$ receptor-dependent transmission (Ben-Ari et al., 1989; Owens et al., 1996) and disappear at around the beginning of the second postnatal week, when GABA actions shift from mainly excitatory to mainly inhibitory (Yamada et al., 2004; Tyzio et al., 2007). To investigate whether a similar developmental profile of NB generation exists in the CA1 in vivo, we next determined the time-course of the fraction of active cells $\Phi(t)$ (Fig. 3A). We found that, at P4, CA1 PCs spent relatively long time periods in a low-activity (silent) state, which was interspersed by transient periods of co-activation, i.e. NBs (Fig. 3A, left). During NBs, $\Phi(t)$ rarely exceeded $20 \%$ indicating that the degree of synchronous activation in vivo is considerably lower than that reported for GDPs in vitro (Leinekugel et al., 1997; Garaschuk et al., 1998; Flossmann et al., 2019). In contrast to GDPs in vitro, bursting activity was even more pronounced at P11, when the network tended to oscillate between an almost silent and 
a bursting state with an inter-burst period of $\sim 2-10$ seconds (Fig. 3A, middle). CA1 PCs frequently maintained such oscillatory behavior for several minutes. At P18, network activity was more continuous than at earlier stages, and large NBs were generally rare (Fig. 3A, right).

To quantify developmental changes in the rhythmicity of network activity, we first computed the power spectrum of $\Phi(\mathrm{t})$. At $\mathrm{P} 11$, this revealed a distinct peak in the range of $\sim 0.1-0.5 \mathrm{~Hz}$ (Fig. 3B), pointing to the existence of a preferred oscillation frequency of CA1 PCs. Such a power peak was absent at P4 and reduced at P18. Accordingly, band-power in the 0.1-0.5 Hz frequency range was significantly higher in the second postnatal week than at earlier or later stages (Fig. 3C, see Table S3).

To characterize periods of synchronized network activity in more detail, we next defined NBs by thresholding $\Phi(\mathrm{t})$ (Fig. 3A and Methods). The fraction of time that the network spent in NBs was lowest at P4 and peaked at P11 (Fig. 3D). Moreover, NBs at P18 were significantly shorter in duration than during the first and second postnatal weeks (Fig. 3E). We quantified NB size as the fraction of active cells (corrected for the threshold applied to $\Phi(t)$ ) and found that it only declined after P11 (Fig. 3F). At P11, each neuron participated in $14.4 \pm 1.2 \%$ of all NBs, which significantly exceeded participation rates at P4 (10.3 \pm 0.8\%) and P18 (11.2 \pm 0.4\%) (see \#5 in Table S3). Additionally, distributions of participation rates were very narrow at P11 (Fig. 3G), pointing to a greater similarity of cells with respect to their contribution to NB generation as compared to earlier or later developmental stages. We confirmed this equalization of the single-neuron contribution to NBs by analyzing the Gini coefficient of participation rates, which we found to be lowest at P11 (\#6 in Table S3).

Taken together, our data reveal that CA1 undergoes a transient period of enhanced bursting activity during the second postnatal week in vivo. These network discharges display 
rhythmicity in the sub-Hz range - in spite of the close-to-random firing of individual PCs.

Enhanced population coupling underlies network burstiness in the second postnatal week in vivo

The transient developmental increase in bursting propensity was unexpected, as (1) GDPs in vitro disappear soon after the first postnatal week (Ben-Ari et al., 1989; Garaschuk et al., 1998; Khazipov et al., 2004) and (2) previous in vivo data from the visual and somatosensory neocortex revealed a desynchronization in firing of local neuronal populations during the same time period (Rochefort et al., 2009; Golshani et al., 2009; van der Bourg et al., 2017; Colonnese et al., 2010). We therefore assessed whether the enhanced burstiness at P11 reflects an increase in functional neuronal coupling. To this end, we first investigated the coupling of single cell firing to that of the overall population. For each cell, we computed its population coupling (PopC) index (Okun et al., 2015; Sweeney et al., 2020) and tested for its significance using surrogate data (see Methods). The PopC index significantly peaked at P11 (Fig. 4A, Table S4), while there was no difference between P4 and P18. The higher PopC index at P11 arose from a significantly higher fraction of coupled cells (Fig. 4B), whereas the indices of coupled cells were similar (Fig. 4C). We next addressed whether the increased PopC index at P11 results from an increase in pairwise temporal correlation of neuronal firing activities. To this end, we computed the spike time tiling coefficient (STTC) as a frequencyindependent affinity metric of two firing-event time series (Cutts et al., 2014) (Fig. 4D). The fraction of significantly correlated cell pairs did not significantly differ between P4 and P11 (P4: $13 \pm 2 \%, P 11: 23 \pm 5 \%$, but strongly decreased to $5 \pm 1 \%$ at P18 (Fig. 4E). Remarkably, STTCs of significantly correlated pairs profoundly declined from $0.18 \pm 0.01$ at P4 to $0.09 \pm$ 0.01 already at $\mathrm{P} 11$, but did not significantly change afterwards (P18: $0.10 \pm 0.00$; Figs. 4F 
and 4G). These data suggest that developmental changes in pairwise neuronal correlations do not account for the increased PopC nor the increased burstiness of CA1 during the second postnatal week. It is worth noting that pairwise correlations of CA1 PCs found here are considerably lower than previously reported for the neonatal neocortex (Golshani et al., 2009). This prompted us to analyze the spatial structure of CA1 ensemble dynamics. We found that the dependence of STTCs on the Euclidean somatic distance was weak already during the first two postnatal weeks and non-significant at P18 (Fig. 4H), indicating that the horizontal confinement of patterned network activity is weak or absent in CA1.

Collectively, our data reveal that enhanced network burstiness during the second postnatal week is associated with a higher fraction of cells being significantly locked to the activity of the local network, while pairwise neuronal correlations are low.

\section{Motifs of CA1 network activity undergo distinct developmental alterations}

Recurring spatiotemporal cellular activation patterns are a hallmark of network activity in the adult hippocampus in vivo (Villette et al., 2015). Whether such repeating patterns (hereafter referred to as 'motifs') are already present at early developmental stages is unknown. To detect motifs, we divided the recording time into non-overlapping bins, each represented by a binary spatial pattern (vector) of active and inactive cells, followed by computing the matching index matrix of all possible pattern pairs (Fig. 5A). We then applied an eigendecompositionbased clustering method to each similarity matrix in order to detect potential motifs, while testing for their significance using surrogate data (see Methods). First, this analysis revealed that the global similarity of the activation patterns was lowest at P11 (Fig. 5B, Table S5), whereas it was similar between $\mathrm{P} 4$ and $\mathrm{P} 18$. This finding implies that there is less commonality between the sets of active cells present in different patterns at $\mathrm{P} 11$, and thus a 
more random recruitment of cells. Furthermore, we found that the number of motifs was significantly lower at P11 (2.5 \pm 0.9$)$ as compared to P4 (5.9 \pm 0.4$)$ and P18 (7.0 \pm 1.1$)$ (Fig. 5C). When computing the fraction of patterns belonging to each motif, we found that the motifs had the highest repetition rate at P18 $(32.0 \pm 4.7 \%)$, while there was no significant difference between P4 (17.8 $\pm 1.2 \%)$ and P11 (16.6 \pm 5.9\%) (Fig. 5D). Taken together, these results suggest that recurring cellular activation patterns become more stable only after the onset of environmental exploration.

A neural network model with inhibitory GABA identifies intrinsic instability dynamics as a key to the emergence of network bursts

Hitherto our analyses of experimental data revealed an unexpected bursting behavior of CA1 PCs at P11, despite the developmental emergence of synaptic inhibition (Tyzio et al., 2007; Murata et al., 2020; Spoljaric et al., 2017), which we found to associate with their higher coupling to local network activity. However, the mechanisms governing in vivo network burstiness as well as its functional implications remain to be understood. Here, we provide mechanistic insights into these open questions by using computational network modeling and stability analysis techniques.

We employed a recurrent neural network (RNN) model of mean firing-activity rates of excitatory glutamatergic (PC) and inhibitory GABAergic (IN) cell populations $\left(A_{\mathrm{p}}\right.$ and $\left.A_{\mathrm{I}}\right)$ with dynamic synaptic weights (Rahmati et al., 2017; Flossmann et al., 2019) (Fig. 6A). Here, we constrain the model with previously reported and our present experimental data obtained for P11: I) GABAergic synapses are considered to be inhibitory (Kirmse et al., 2015; Valeeva et al., 2016; Murata et al., 2020) and II) the spontaneous time-averaged $A_{\mathrm{p}}$ is effectively nonzero (Fig. 2C). We found that such a network operates under a bi-stable regime, where two 
stable spontaneous fixed points (FPs) exist: one at a silent state $\left(A_{\mathrm{P}}=A_{I}=0 \mathrm{~Hz}\right)$ and the other at an active state $\left(A_{\mathrm{p}} \neq 0 \mathrm{~Hz}\right.$ and $A_{\mathrm{I}} \neq 0 \mathrm{~Hz}$; green dots in Fig. 6B). The ability of the network to embed the latter FP is mainly due to the stabilization function of inhibitory GABA (Rahmati et al., 2017; Latham et al., 2004). Strikingly, our simulations showed that the network can process a given input quite differently at the silent and active states, respectively (time points $\mathrm{a}$ and $\mathrm{c}$ in Fig. 6C). To this end, we applied a set of two excitatory inputs to the network's PC and IN populations ( $e_{\mathrm{P}}$ and $e_{\mathrm{I}}$ ), resembling e.g. SPW-driven inputs to CA1 (Fig. 6C). We set the input strengths and duration to be identical across the two states. We found that, when operating at the active state, the network activity monotonically decays back to this state, once the input ceases (Fig. 6C, a). However, at the silent state, input removal is followed by a transient profound surge in network activity (c in Fig. 6C). Hereafter, we refer to this supra-amplification activity as simulated NB (simNB), emulating experimentally observed NBs (Fig. 3).

\section{Network state-dependency of simNB generation}

To disclose the mechanisms underlying this distinct behavior of the network at the silent and active state (Fig. 6C), we computed the corresponding steady-state $A_{1}-A_{\mathrm{p}}$-plane of the network, after freezing the slow short-term synaptic plasticity (STP) dynamics and, thus, synaptic weights (Frozen STP-RNNs), at either of these states separately (Fig. 6D). This analysis enables assessing the initial phase of network activity following an input perturbation. We found that, while operating at the silent state, the active state is not initially accessible to the network (lower panel in Fig. 6D). Instead, an unstable FP is present in the network's fast (i.e. firing activity) dynamics, which builds an amplification threshold around the attraction domain of the FP located at the silent state. This in turn allows for the emergence of simNBs: 
A sufficiently strong perturbation, amenable to initially push the network activity beyond this threshold (i.e. to the amplification domain), will transiently expose the network to its intrinsic instability-driven dynamics, thereby effectively triggering a simNB (Fig. 6C, c). Note that this unstable FP is different from its counterpart in the full system (Fig. 6B) and is only visible in the network's fast dynamics. In particular, this FP is transient and disappears around the peak of the elicited simNB, mainly due to short-term synaptic depression (Rahmati et al., 2017). Unlike the silent state, the network frozen at the active state has no amplification domain, but instead two attraction domains pertaining to its FPs at silent and active states (upper panel in Fig. 6D). This explains the network's incapability of eliciting simNBs, when operating at the active state. Collectively, these results suggest that simNBs, initiated by the input, are mainly an expression of the network's intrinsic instability dynamics, where the silent periods of the network are a prerequisite for its emergence.

Input-strength dependency and internal deadline of state transitions

What are the input requirements that allow the network to transition between the active and the silent states? First, we found that the silencing of the network in an active state requires specific ratios of excitatory input strengths to be delivered to its PC and IN populations (Figs. $6 E-G)$. In particular, the presence of GABAergic inhibition can effectively promote this transition, where otherwise a relatively much stronger $e_{\mathrm{p}}$ is required to silence the network solely (Fig. 6G). Furthermore, once silenced, pushing the network back to the active state is also dependent on input ratio (Figs. $6 \mathrm{H}-\mathrm{J}$ ). However, to make such a transition, the network becomes noticeably more selective about the input ratio (compare Figs. $6 \mathrm{G}$ and $6 \mathrm{~J}$ ). Besides, for both transitions, the proper ratios of the inputs are effectively determined by the approximated initial phase of the network response (Fig. 6D), and thus mainly dependent on 
the synaptic weights right before the input arrival. In sum, these results suggest that proper input strengths onto the PC and IN populations, along with the inhibitory action of GABA, play key roles in the dynamic state transitioning of the network, thereby allowing for its burstiness. Considering the dynamics of synaptic weights in our model along with their significance for state transitions, we next investigated the impact of input timing (Fig. 7). Furthermore, we found that, once silenced by the first input, a deadline is formed for the network's transitioning back to the active state (dotted line in Figs. 7A, D, G, H). If the second input misses the deadline, the network will elicit a large-amplitude simNB, which is not able to converge to the active state any longer (Fig. 7D). Prior to this deadline and depending on the input ratio (Fig. $6 \mathrm{~J}$ ), the network will either transition to the active state (Figs. $7 \mathrm{~A}, 6 \mathrm{H}$ ) or return to the silent state (Fig. 6I). Importantly, our analysis showed that this deadline is an internal property of the network and cannot be overruled by any input level (see below). Therefore, specific combinations of input ratio (Fig. 6J) and input timing (Fig. 7G) are required for transitioning to the active state. In addition, once the simNB failed to converge to the active state, the network will encounter a new deadline (see Fig. S1). In sum, these results imply that the silent state of the network can have per se different hidden sub-states, each with a specific input-encoding operating scheme.

Having found the intrinsic deadline as a main determinant for the type of network burst, we next investigated the origin of these different activity patterns: How does the network decide between transitioning to the active state and returning to the silent state? Remarkably, we found that the deadline for network transitioning to the active state is mechanistically dependent on the presence of a transient stable FP in its fast dynamics around the peak of the simNB. This can be seen in the two examples where the network receives the same input but at different inter-pulse intervals (IPIS), one preceding (Figs. 7A-C) and the other 
exceeding the deadline (Figs. 7D-F). For both IPIS, at the time right before the second input (Figs. 7B, E), the Frozen RNNs only provide evidence for the emergence of simNB, but not for the state transition (note the presence of an amplification domain; pink area). Importantly, we found, however, that in the case of the shorter IPI, the network is able to form a transient, stable non-zero FP in its Frozen RNN, at the peak of the simNB (compare Figs. 7C and 7F). This FP can transiently attract the network's activity towards itself, and as the activity evolves accordingly, it also changes its position in the corresponding updated Frozen STP-RNN, until eventually converging to its counterpart in the full system. Intuitively, this transient, stable FP can guide the network's activity towards that of the full system (see the non-origin green dot in Fig. 6B). The temporal repositioning of this stable FP is due to the activity-dependency of the synaptic weights in our model. Besides, our findings show that the existence of this FP around the simNB peak is effectively determined by simNB size (Fig. 7G). If simNB size exceeds an internally determined threshold, the network cannot build such a transient stable FP due to a reduction of synaptic weights (Rahmati et al., 2017); consequently, the simNB will be attracted towards the silent state. In this line, Fig. 7G shows that simNB size is effectively determined by the IPI: The longer the IPI (thus, the silent period) is, the larger the simNB will be. Here, the IPI-dependency of the simNB size mainly reflects the slow recovery from shortterm depression of excitatory synapses at the silent state (Fig. 7H).

In conclusion, our modeling results indicate that developing CA1 possesses multiple inputencoding schemes, which are effectively determined by three factors: 1) the input ratio, 2) the input timing, and 3) the non-linearity and dynamics of synaptic weights. 


\section{Discussion}

Unique characteristics of network dynamics in developing CA1 in vivo

Using in vivo two-photon $\mathrm{Ca}^{2+}$ imaging, we here reveal that cellular network dynamics in developing hippocampal CA1 differ from those previously observed in neocortical areas. Firstly, pairwise correlations of firing activities (Figs. 4D-G) were considerably lower than those in the visual (Rochefort et al., 2009) or somatosensory (Golshani et al., 2009; Che et al., 2018; van der Bourg et al., 2017) cortex in vivo, pointing to a lower degree of neuronal synchrony in CA1. Similarly, the degree of neuronal co-activation was substantially lower than that during GDPs recorded in acute slices (Flossmann et al., 2019), supporting the view that the underlying dynamics differ markedly. Secondly, throughout the developmental period studied here, pairwise correlations of firing activity were only weakly dependent on the intersomatic distance (Fig. 4H). Together with previous electrophysiological data demonstrating bilateral synchronization of CA1 MUA along the septal-temporal axis (Valeeva et al., 2020; Valeeva et al., 2019), these observations imply that network activity in the neonatal CA1 is less correlated on small, but more correlated on large spatial scales, as compared to neocortical areas. In other words, CA1 NBs tend to lack a sharp horizontal confinement typical of neocortical spindle bursts, which activate upper layer PCs in a columnar manner (Kummer et al., 2016; Kirmse et al., 2015). Wavefront-containing activity patterns appear to be necessary for the proper developmental refinement of topographic maps in neocortex (Cang et al., 2005; Li et al., 2013) and receptive field characteristics of visual cortical neurons (Albert et al., 2008). In this line, the peculiar spatial features of CA1 NBs in our data may reflect the absence of a clear topical macro-organization of the mature hippocampus (Bellistri et al., 2013). 
A role for intrinsic network instability and synaptic inhibition in the generation of synchronized network activity in CA1

We demonstrate that CA1 PCs undergo a transient period of enhanced network synchrony in the second postnatal week, i.e. shortly before the onsets of pattern vision, active whisking and environmental exploration. This trajectory remarkably differs from what has been previously reported for the hippocampus in vitro, where GDPs disappear shortly after the first postnatal week. At this time, GABA-releasing INs already impose effective synaptic inhibition on CA1 PCs (Tyzio et al., 2008; Spoljaric et al., 2017; Murata et al., 2020), implying that synchronized activity in vivo does not depend on a GABAergic excitatory drive (in contrast to GDPs). Synchronized CA1 activity in the second postnatal week exhibited a preferred frequency of -0.1-0.5 Hz, indicating that NBs occur in a temporally non-random manner (Fig. 3). Strikingly, however, individual neurons were recruited more randomly at this age, as the number of significant motifs of network activity as well as their average repetition probability were lowest (Fig. 5). In addition, the firing of individual cells resembled a Poissonian process (Fig. 2), and pairwise neuronal correlations were lowest in the second postnatal week (Fig. 4). We here set out to explain these seemingly discordant experimental findings using data-informed computational modeling.

Capitalizing on a dynamic systems modeling approach, we show that a potential dynamical regime of the network that allows for the generation of synchronized activity in the presence of effective synaptic inhibition is bi-stability. We found that our network model is prone to an intrinsic instability, governed by a nonlinear interaction between its fast (firing) and slow (synaptic) dynamics. Such instability enables the model to over-amplify the input, even after its removal, and thus elicit network bursts (simNBs; Fig. 6). This indicates that a (sim)NB reflects a spatiotemporal trajectory of the network's intrinsic instability dynamics, which, due 
to its nature, can recruit a random set of cells at random order within a specific time-window. The size of this set and the time-window are determined by the synaptic weights right before the input arrival (Rahmati et al., 2017). Importantly, the data-informed model mechanistically links strong population coupling to weak pairwise neuronal correlations, the close-to-random firing of individual PCs and the low number of network motifs - as we found experimentally for the second postnatal week.

What are the functional roles of burstiness and synaptic inhibition at this stage? Our model, in addition to its silent state, embeds a stable fixed point (or steady state) at non-zero low activity rates (Fig. 6B), in accordance with our recorded data. Theoretical studies showed that the presence of such a fixed point requires the stabilization function of inhibitory GABA (Rahmati et al., 2017; Latham et al., 2004; Ozeki et al., 2009; Tsodyks et al., 1997). At such a fixed point, the network can operate under an inhibition-stabilized regime, which may enable CA1 networks to begin performing complex computations (Latham et al., 2004; Tsodyks et al., 1997). The ability of the network to dynamically transition between its silent and active states in an input-dependent fashion (Fig. 6) renders the second postnatal week an early developmental stage toward forming hippocampal memory and cognition mechanisms, as found in adult hippocampal attractor networks (Rolls, 2007; Knierim et al., 2016; Hartley et al., 2014; see also Rahmati et al., 2017). This view is supported by (I) the existence of the internal deadlines as well as a delicate input-ratio and -timing dependency of successful state transitions and simNB generation and (II) the network's ability to store information in both the silent and active state through transient synaptic weights (Mongillo et al., 2008; Stokes, 2015; Barak et al., 2014) and persistent activity (Boran et al., 2019; Zylberberg et al., 2017), respectively. In this line, our modeling results further imply that the network's silent state has per se several dynamic operational sub-states, which keep track of input timing and 
strength (Figs. 7 and S1) to produce proper network read-outs. Collectively, we postulate that the basis of CA1 encoding schemes is set in shortly before eye opening. Moreover, our data suggest that GDPs disappear due to improper synaptic inputs in vitro during the second postnatal week, when GABA actions switch to inhibitory (Murata et al., 2020; Valeeva et al., 2016).

\section{Potential developmental functions of network bursts in the neonatal CA1}

Computational modeling suggests a mechanism, whereby CA1 undergoes extensive inputdiscrimination learning before eye opening. In this scenario, NBs serve as a feedback that informs individual CA1 PCs about functionally important characteristics of the synaptic input to the local network, including (I) the proper targeting ratio of excitatory PCs versus inhibitory GABAergic INs (Murata et al., 2020; Valeeva et al., 2016) and (II) the timing of inputs relative to the network's operational state. Interestingly, the developmental period of enhanced network burstiness coincides with a major surge of synaptogenesis in CA1 PCs (Kirov et al., 2004). The latter involves a net addition of synapses, but also functionally important anatomical rearrangements. Specifically, the formation of mature dendritic spines, which allow for electrical and metabolic compartmentalization of postsynaptic responses, commences only at around $\mathrm{P} 10$, by which time most glutamatergic synapses are rather localized to dendritic shafts (Fiala et al., 1998; Kirov et al., 2004). In addition to acting as potential synaptogenic stimuli (Kirov et al., 2004), NBs could thus be an important element underlying synaptic competition and pruning, for example, based on synchronization-dependent plasticity rules in nascent dendrites (Winnubst et al., 2015). Synchronized activity might therefore be causally related to the delayed development of skewed (approximately log-normal) firing rate distributions (Fig. 2) underlying sparse coding (Ikegaya et al., 2013; Yassin et al., 2010; 
469 Trojanowski et al., 2020; Narayanan et al., 2012; Roxin et al., 2011) - an energy-efficient

470 regime of input processing and information storage (Mizuseki et al., 2013). In accordance with

471 the efficient coding hypothesis and seminal work in the visual system (Albert et al., 2008), we

472 argue that one function of developing CA1 and, thus, NBs is to remove statistical redundancy

473 in the multi-sensory place-field code, by making use of a learning scheme that uses both

474 intrinsically and sensory-evoked activity already before environmental exploration. 


\section{Acknowledgments}

476 We thank Ina Ingrisch for excellent technical assistance. This work was supported by

477 Individual Research Grants (KI 1816/6-1, KI 1816/7-1 to K.K., HO 2156/5-1, HO 2156/6-1 to

478 K.H.), the Research Unit 3004 (KI 1816/5-1 to K.K., GE 2519/8-1, GE 2519/9-1 to C.G.), the

479 Priority Program 1665 (HO 2156/3-1/2 to K.H., KI 1816/1-1/2 to K.K., KI 1638/3-1/2 to

480 S.J.K.) and the CRC Transregio 166 (B2 to C.G., B3 to K.H., K.K.) of the German Research 481 Foundation. 
483 Conceptualization, K.K., K.H., V.R.; Methodology, J.G., K.K., V.R.; Formal Analysis, K.K.,

484 J.G., V.R.; Investigation, J.G., K.K., V.R., M.M.; Computational modeling, V.R.; Writing -

485 Original Draft, K.K., V.R., J.G.; Writing - Review \& Editing, J.G., V.R., M.M., O.W.W., C.G.,

486 S.J.K., K.H., K.K.; Supervision, K.K., K.H., O.W.W., S.J.K., C.G.; Funding Acquisition, K.K., 487 K.H., O.W.W., S.J.K., C.G. 
bioRxiv preprint doi: https://doi.org/10.1101/2021.05.28.446133; this version posted May 29, 2021. The copyright holder for this preprint (which was not certified by peer review) is the author/funder. All rights reserved. No reuse allowed without permission.

\section{Declaration of Interests}

489 The authors declare no competing interests. 


\section{References}

Albert, M.V., Schnabel, A., and Field, D.J. (2008). Innate visual learning through spontaneous activity patterns. PLoS Comput Biol 4, e1000137.

Barak, O., and Tsodyks, M. (2014). Working models of working memory. Curr Opin Neurobiol 25, 20-24.

Bellistri, E., Aguilar, J., Brotons-Mas, J.R., Foffani, G., and de la Prida, L.M. (2013). Basic properties of somatosensory-evoked responses in the dorsal hippocampus of the rat. $\mathrm{J}$ Physiol 591, 2667-2686.

Ben-Ari, Y., Cherubini, E., Corradetti, R., and Gaiarsa, J.L. (1989). Giant synaptic potentials in immature rat CA3 hippocampal neurones. J Physiol 416, 303-325.

Blanquie, O., Yang, J.W., Kilb, W., Sharopov, S., Sinning, A., and Luhmann, H.J. (2017). Electrical activity controls area-specific expression of neuronal apoptosis in the mouse developing cerebral cortex. Elife 6, e27696.

Boran, E., Fedele, T., Klaver, P., Hilfiker, P., Stieglitz, L., Grunwald, T., and Sarnthein, J. (2019). Persistent hippocampal neural firing and hippocampal-cortical coupling predict verbal working memory load. Sci Adv 5, eaav3687.

Cang, J., Renteria, R.C., Kaneko, M., Liu, X., Copenhagen, D.R., and Stryker, M.P. (2005). Development of precise maps in visual cortex requires patterned spontaneous activity in the retina. Neuron 48, 797-809.

Che, A., Babij, R., lannone, A.F., Fetcho, R.N., Ferrer, M., Liston, C., Fishell, G., and De Marco Garcia, N.V. (2018). Layer I Interneurons Sharpen Sensory Maps during Neonatal Development. Neuron 99, 98-116 e117.

Chen, Y., Jang, H., Spratt, P.W.E., Kosar, S., Taylor, D.E., Essner, R.A., Bai, L., Leib, D.E., Kuo, T.W., Lin, Y.C., et al. (2020). Soma-Targeted Imaging of Neural Circuits by Ribosome Tethering. Neuron 107, 454-469 e456.

Clements, J.D., and Bekkers, J.M. (1997). Detection of spontaneous synaptic events with an optimally scaled template. Biophys J 73, 220-229.

Colonnese, M.T., Kaminska, A., Minlebaev, M., Milh, M., Bloem, B., Lescure, S., Moriette, G., Chiron, C., Ben-Ari, Y., and Khazipov, R. (2010). A conserved switch in sensory processing prepares developing neocortex for vision. Neuron 67, 480-498.

Cutts, C.S., and Eglen, S.J. (2014). Detecting pairwise correlations in spike trains: an objective comparison of methods and application to the study of retinal waves. The Journal of neuroscience : the official journal of the Society for Neuroscience 34, 14288-14303.

Del Rio-Bermudez, C., Kim, J., Sokoloff, G., and Blumberg, M.S. (2020). Active Sleep Promotes Coherent Oscillatory Activity in the Cortico-Hippocampal System of Infant Rats. Cereb Cortex 30, 2070-2082. 
Denis, J., Dard, R.F., Quiroli, E., Cossart, R., and Picardo, M.A. (2020). DeepCINAC: A DeepLearning-Based Python Toolbox for Inferring Calcium Imaging Neuronal Activity Based on Movie Visualization. eNeuro 7.

Dzhala, V.I., Talos, D.M., Sdrulla, D.A., Brumback, A.C., Mathews, G.C., Benke, T.A., Delpire, E., Jensen, F.E., and Staley, K.J. (2005). NKCC1 transporter facilitates seizures in the developing brain. Nat Med 11, 1205-1213.

Fiala, J.C., Feinberg, M., Popov, V., and Harris, K.M. (1998). Synaptogenesis via dendritic filopodia in developing hippocampal area CA1. The Journal of neuroscience : the official journal of the Society for Neuroscience 18, 8900-8911.

Flossmann, T., Kaas, T., Rahmati, V., Kiebel, S.J., Witte, O.W., Holthoff, K., and Kirmse, K. (2019). Somatostatin Interneurons Promote Neuronal Synchrony in the Neonatal Hippocampus. Cell Rep 26, 3173-3182.

Garaschuk, O., Hanse, E., and Konnerth, A. (1998). Developmental profile and synaptic origin of early network oscillations in the CA1 region of rat neonatal hippocampus. J Physiol 507 ( $P t$ 1), 219-236.

Golshani, P., Goncalves, J.T., Khoshkhoo, S., Mostany, R., Smirnakis, S., and PorteraCailliau, C. (2009). Internally mediated developmental desynchronization of neocortical network activity. The Journal of neuroscience : the official journal of the Society for Neuroscience 29, 10890-10899.

Gorski, J.A., Talley, T., Qiu, M., Puelles, L., Rubenstein, J.L., and Jones, K.R. (2002). Cortical excitatory neurons and glia, but not GABAergic neurons, are produced in the Emx1expressing lineage. The Journal of neuroscience : the official journal of the Society for Neuroscience 22, 6309-6314.

Graf, J., Zhang, C., Marguet, S.L., Herrmann, T., Flossmann, T., Hinsch, R., Rahmati, V., Guenther, M., Frahm, C., Urbach, A., et al. (2021). A limited role of NKCC1 in telencephalic glutamatergic neurons for developing hippocampal network dynamics and behavior. Proc Natl Acad Sci U S A 118.

Gretenkord, S., Kostka, J.K., Hartung, H., Watznauer, K., Fleck, D., Minier-Toribio, A., Spehr, M., and Hanganu-Opatz, I.L. (2019). Coordinated electrical activity in the olfactory bulb gates the oscillatory entrainment of entorhinal networks in neonatal mice. PLoS Biol 17, e2006994.

Hartley, T., Lever, C., Burgess, N., and O'Keefe, J. (2014). Space in the brain: how the hippocampal formation supports spatial cognition. Philos Trans R Soc Lond B Biol Sci 369, 20120510.

Holt, G.R., Softky, W.R., Koch, C., and Douglas, R.J. (1996). Comparison of discharge variability in vitro and in vivo in cat visual cortex neurons. J Neurophysiol 75, 1806-1814.

Ikegaya, Y., Sasaki, T., Ishikawa, D., Honma, N., Tao, K., Takahashi, N., Minamisawa, G., Ujita, S., and Matsuki, N. (2013). Interpyramid spike transmission stabilizes the sparseness of recurrent network activity. Cereb Cortex 23, 293-304. 
Karlsson, K.A., Mohns, E.J., di Prisco, G.V., and Blumberg, M.S. (2006). On the cooccurrence of startles and hippocampal sharp waves in newborn rats. Hippocampus 16, 959965.

Kerr, J.N., Greenberg, D., and Helmchen, F. (2005). Imaging input and output of neocortical networks in vivo. Proc Natl Acad Sci U S A 102, 14063-14068.

Khazipov, R., Khalilov, I., Tyzio, R., Morozova, E., Ben-Ari, Y., and Holmes, G.L. (2004). Developmental changes in GABAergic actions and seizure susceptibility in the rat hippocampus. The European journal of neuroscience 19, 590-600.

Kirkby, L.A., Sack, G.S., Firl, A., and Feller, M.B. (2013). A role for correlated spontaneous activity in the assembly of neural circuits. Neuron 80, 1129-1144.

Kirmse, K., Kummer, M., Kovalchuk, Y., Witte, O.W., Garaschuk, O., and Holthoff, K. (2015). GABA depolarizes immature neurons and inhibits network activity in the neonatal neocortex in vivo. Nature communications 6, 7750.

Kirov, S.A., Goddard, C.A., and Harris, K.M. (2004). Age-dependence in the homeostatic upregulation of hippocampal dendritic spine number during blocked synaptic transmission. Neuropharmacology 47, 640-648.

Knierim, J.J., and Neunuebel, J.P. (2016). Tracking the flow of hippocampal computation: Pattern separation, pattern completion, and attractor dynamics. Neurobiol Learn Mem 129, 38-49.

Kruskal, P.B., Stanis, J.J., McNaughton, B.L., and Thomas, P.J. (2007). A binless correlation measure reduces the variability of memory reactivation estimates. Stat Med 26, 3997-4008.

Kummer, M., Kirmse, K., Witte, O.W., Haueisen, J., and Holthoff, K. (2015). Method to quantify accuracy of position feedback signals of a three-dimensional two-photon laserscanning microscope. Biomed Opt Express 6, 3678-3693.

Kummer, M., Kirmse, K., Witte, O.W., and Holthoff, K. (2012). Reliable in vivo identification of both GABAergic and glutamatergic neurons using Emx1-Cre driven fluorescent reporter expression. Cell Calcium 52, 182-189.

Kummer, M., Kirmse, K., Zhang, C., Haueisen, J., Witte, O.W., and Holthoff, K. (2016). Column-like $\mathrm{Ca}(2+)$ clusters in the mouse neonatal neocortex revealed by three-dimensional two-photon $\mathrm{Ca}(2+)$ imaging in vivo. Neurolmage 138, 64-75.

Latham, P.E., and Nirenberg, S. (2004). Computing and stability in cortical networks. Neural Comput 16, 1385-1412.

Leinekugel, X., Khazipov, R., Cannon, R., Hirase, H., Ben-Ari, Y., and Buzsaki, G. (2002). Correlated bursts of activity in the neonatal hippocampus in vivo. Science 296, 2049-2052.

Leinekugel, X., Medina, I., Khalilov, I., Ben-Ari, Y., and Khazipov, R. (1997). Ca2+ oscillations mediated by the synergistic excitatory actions of $\operatorname{GABA}(A)$ and NMDA receptors in the neonatal hippocampus. Neuron 18, 243-255. 
Li, H., Fertuzinhos, S., Mohns, E., Hnasko, T.S., Verhage, M., Edwards, R., Sestan, N., and Crair, M.C. (2013). Laminar and columnar development of barrel cortex relies on thalamocortical neurotransmission. Neuron 79, 970-986.

Li, X., Ouyang, G., Usami, A., Ikegaya, Y., and Sik, A. (2010). Scale-free topology of the CA3 hippocampal network: a novel method to analyze functional neuronal assemblies. Biophys $\mathrm{J}$ 98, 1733-1741.

Maset, A., Galla, L., Francia, S., Cozzolino, O., Capasso, P., Goisis, R.C., Losi, G., Lombardo, A., Ratto, G.M., and Lodovichi, C. (2021). Altered Cl(-) homeostasis hinders forebrain GABAergic interneuron migration in a mouse model of intellectual disability. Proc Natl Acad Sci U S A 118.

Mizrahi, A., Crowley, J.C., Shtoyerman, E., and Katz, L.C. (2004). High-resolution in vivo imaging of hippocampal dendrites and spines. The Journal of neuroscience : the official journal of the Society for Neuroscience 24, 3147-3151.

Mizuseki, K., and Buzsaki, G. (2013). Preconfigured, skewed distribution of firing rates in the hippocampus and entorhinal cortex. Cell Rep 4, 1010-1021.

Molnar, Z., Luhmann, H.J., and Kanold, P.O. (2020). Transient cortical circuits match spontaneous and sensory-driven activity during development. Science 370.

Mongillo, G., Barak, O., and Tsodyks, M. (2008). Synaptic theory of working memory. Science 319, 1543-1546.

Murata, Y., and Colonnese, M.T. (2020). GABAergic interneurons excite neonatal hippocampus in vivo. Science Advances 6.

Narayanan, R., and Johnston, D. (2012). Functional maps within a single neuron. J Neurophysiol 108, 2343-2351.

Nguyen, Q.T., Tsai, P.S., and Kleinfeld, D. (2006). MPScope: a versatile software suite for multiphoton microscopy. J Neurosci Methods 156, 351-359.

Oh, W.C., Lutzu, S., Castillo, P.E., and Kwon, H.B. (2016). De novo synaptogenesis induced by GABA in the developing mouse cortex. Science 353, 1037-1040.

Okun, M., Steinmetz, N., Cossell, L., lacaruso, M.F., Ko, H., Bartho, P., Moore, T., Hofer, S.B., Mrsic-Flogel, T.D., Carandini, M., and Harris, K.D. (2015). Diverse coupling of neurons to populations in sensory cortex. Nature 521, 511-515.

Owens, D.F., Boyce, L.H., Davis, M.B., and Kriegstein, A.R. (1996). Excitatory GABA responses in embryonic and neonatal cortical slices demonstrated by gramicidin perforatedpatch recordings and calcium imaging. The Journal of neuroscience : the official journal of the Society for Neuroscience 16, 6414-6423.

Ozeki, H., Finn, I.M., Schaffer, E.S., Miller, K.D., and Ferster, D. (2009). Inhibitory stabilization of the cortical network underlies visual surround suppression. Neuron 62, 578-592. 
Patel, T.P., Man, K., Firestein, B.L., and Meaney, D.F. (2015). Automated quantification of neuronal networks and single-cell calcium dynamics using calcium imaging. J Neurosci Methods 243, 26-38.

Pnevmatikakis, E.A., and Giovannucci, A. (2017). NoRMCorre: An online algorithm for piecewise rigid motion correction of calcium imaging data. J Neurosci Methods 291, 83-94.

Ponce-Alvarez, A., Kilavik, B.E., and Riehle, A. (2010). Comparison of local measures of spike time irregularity and relating variability to firing rate in motor cortical neurons. J Comput Neurosci 29, 351-365.

Rahmati, V., Kirmse, K., Holthoff, K., and Kiebel, S.J. (2018). Ultra-fast accurate reconstruction of spiking activity from calcium imaging data. J Neurophysiol 119, 1863-1878.

Rahmati, V., Kirmse, K., Holthoff, K., Schwabe, L., and Kiebel, S.J. (2017). Developmental Emergence of Sparse Coding: A Dynamic Systems Approach. Sci Rep 7, 13015.

Rochefort, N.L., Garaschuk, O., Milos, R.I., Narushima, M., Marandi, N., Pichler, B., Kovalchuk, Y., and Konnerth, A. (2009). Sparsification of neuronal activity in the visual cortex at eye-opening. Proc Natl Acad Sci U S A 106, 15049-15054.

Rolls, E.T. (2007). An attractor network in the hippocampus: theory and neurophysiology. Learn Mem 14, 714-731.

Romano, S.A., Pietri, T., Perez-Schuster, V., Jouary, A., Haudrechy, M., and Sumbre, G. (2015). Spontaneous neuronal network dynamics reveal circuit's functional adaptations for behavior. Neuron 85, 1070-1085.

Roxin, A., Brunel, N., Hansel, D., Mongillo, G., and van Vreeswijk, C. (2011). On the distribution of firing rates in networks of cortical neurons. The Journal of neuroscience : the official journal of the Society for Neuroscience 31, 16217-16226.

Sando, R., Bushong, E., Zhu, Y., Huang, M., Considine, C., Phan, S., Ju, S., Uytiepo, M., Ellisman, M., and Maximov, A. (2017). Assembly of Excitatory Synapses in the Absence of Glutamatergic Neurotransmission. Neuron 94, 312-321 e313.

Spoljaric, A., Seja, P., Spoljaric, I., Virtanen, M.A., Lindfors, J., Uvarov, P., Summanen, M., Crow, A.K., Hsueh, B., Puskarjov, M., et al. (2017). Vasopressin excites interneurons to suppress hippocampal network activity across a broad span of brain maturity at birth. Proc Natl Acad Sci U S A 114, E10819-E10828.

Spoljaric, I., Spoljaric, A., Mavrovic, M., Seja, P., Puskarjov, M., and Kaila, K. (2019). KCC2Mediated $\mathrm{Cl}(-)$ Extrusion Modulates Spontaneous Hippocampal Network Events in Perinatal Rats and Mice. Cell Rep 26, 1073-1081 e1073.

Sporns, O., Honey, C.J., and Kotter, R. (2007). Identification and classification of hubs in brain networks. PLoS One 2, e1049.

Stokes, M.G. (2015). 'Activity-silent' working memory in prefrontal cortex: a dynamic coding framework. Trends Cogn Sci 19, 394-405. 
Sun, S., Babola, T., Pregernig, G., So, K.S., Nguyen, M., Su, S.M., Palermo, A.T., Bergles, D.E., Burns, J.C., and Muller, U. (2018). Hair Cell Mechanotransduction Regulates Spontaneous Activity and Spiral Ganglion Subtype Specification in the Auditory System. Cell 174, 1247-1263 e1215.

Sweeney, Y., and Clopath, C. (2020). Population coupling predicts the plasticity of stimulus responses in cortical circuits. Elife 9.

Trojanowski, N.F., Bottorff, J., and Turrigiano, G.G. (2020). Activity labeling in vivo using CaMPARI2 reveals intrinsic and synaptic differences between neurons with high and low firing rate set points. Neuron.

Tsodyks, M., Pawelzik, K., and Markram, H. (1998). Neural networks with dynamic synapses. Neural Comput 10, 821-835.

Tsodyks, M.V., Skaggs, W.E., Sejnowski, T.J., and McNaughton, B.L. (1997). Paradoxical effects of external modulation of inhibitory interneurons. The Journal of neuroscience : the official journal of the Society for Neuroscience 17, 4382-4388.

Tyzio, R., Holmes, G.L., Ben-Ari, Y., and Khazipov, R. (2007). Timing of the developmental switch in GABA(A) mediated signaling from excitation to inhibition in CA3 rat hippocampus using gramicidin perforated patch and extracellular recordings. Epilepsia 48 Suppl 5, 96-105.

Tyzio, R., Minlebaev, M., Rheims, S., Ivanov, A., Jorquera, I., Holmes, G.L., Zilberter, Y., Ben-Ari, Y., and Khazipov, R. (2008). Postnatal changes in somatic gamma-aminobutyric acid signalling in the rat hippocampus. The European journal of neuroscience 27, 2515-2528.

Valeeva, G., Nasretdinov, A., Rychkova, V., and Khazipov, R. (2019). Bilateral Synchronization of Hippocampal Early Sharp Waves in Neonatal Rats. Front Cell Neurosci $13,29$.

Valeeva, G., Rychkova, V., Vinokurova, D., Nasretdinov, A., and Khazipov, R. (2020). Early sharp wave synchronization along the septo-temporal axis of the neonatal rat hippocampus. ЖУРНАЛ ВЫСШЕЙ НЕРВНОЙ ДЕЯТЕЛЬНОСТИ ИМ ИП ПАВЛОВА 70, 341-350.

Valeeva, G., Tressard, T., Mukhtarov, M., Baude, A., and Khazipov, R. (2016). An Optogenetic Approach for Investigation of Excitatory and Inhibitory Network GABA Actions in Mice Expressing Channelrhodopsin-2 in GABAergic Neurons. The Journal of neuroscience : the official journal of the Society for Neuroscience 36, 5961-5973.

van der Bourg, A., Yang, J.W., Reyes-Puerta, V., Laurenczy, B., Wieckhorst, M., Stuttgen, M.C., Luhmann, H.J., and Helmchen, F. (2017). Layer-Specific Refinement of Sensory Coding in Developing Mouse Barrel Cortex. Cereb Cortex 27, 4835-4850.

Villette, V., Malvache, A., Tressard, T., Dupuy, N., and Cossart, R. (2015). Internally Recurring Hippocampal Sequences as a Population Template of Spatiotemporal Information. Neuron 88, 357-366.

Vitali, I., Fievre, S., Telley, L., Oberst, P., Bariselli, S., Frangeul, L., Baumann, N., McMahon, J.J., Klingler, E., Bocchi, R., et al. (2018). Progenitor Hyperpolarization Regulates the 
Sequential Generation of Neuronal Subtypes in the Developing Neocortex. Cell 174, 12641276 e1215.

Winnubst, J., Cheyne, J.E., Niculescu, D., and Lohmann, C. (2015). Spontaneous Activity Drives Local Synaptic Plasticity In Vivo. Neuron 87, 399-410.

Wong, F.K., Bercsenyi, K., Sreenivasan, V., Portales, A., Fernandez-Otero, M., and Marin, O. (2018). Pyramidal cell regulation of interneuron survival sculpts cortical networks. Nature 557, 668-673.

Yamada, J., Okabe, A., Toyoda, H., Kilb, W., Luhmann, H.J., and Fukuda, A. (2004). Cluptake promoting depolarizing GABA actions in immature rat neocortical neurones is mediated by NKCC1. J Physiol 557, 829-841.

Yassin, L., Benedetti, B.L., Jouhanneau, J.S., Wen, J.A., Poulet, J.F., and Barth, A.L. (2010). An embedded subnetwork of highly active neurons in the neocortex. Neuron 68, 1043-1050.

Zhang, C., Yang, S., Flossmann, T., Gao, S., Witte, O.W., Nagel, G., Holthoff, K., and Kirmse, K. (2019). Optimized photo-stimulation of halorhodopsin for long-term neuronal inhibition. BMC Biol 17, 95.

Zhang, J., Ackman, J.B., Xu, H.P., and Crair, M.C. (2011). Visual map development depends on the temporal pattern of binocular activity in mice. Nature neuroscience 15, 298-307.

Zylberberg, J., and Strowbridge, B.W. (2017). Mechanisms of Persistent Activity in Cortical Circuits: Possible Neural Substrates for Working Memory. Annu Rev Neurosci 40, 603-627. 


\section{Figures and legends}

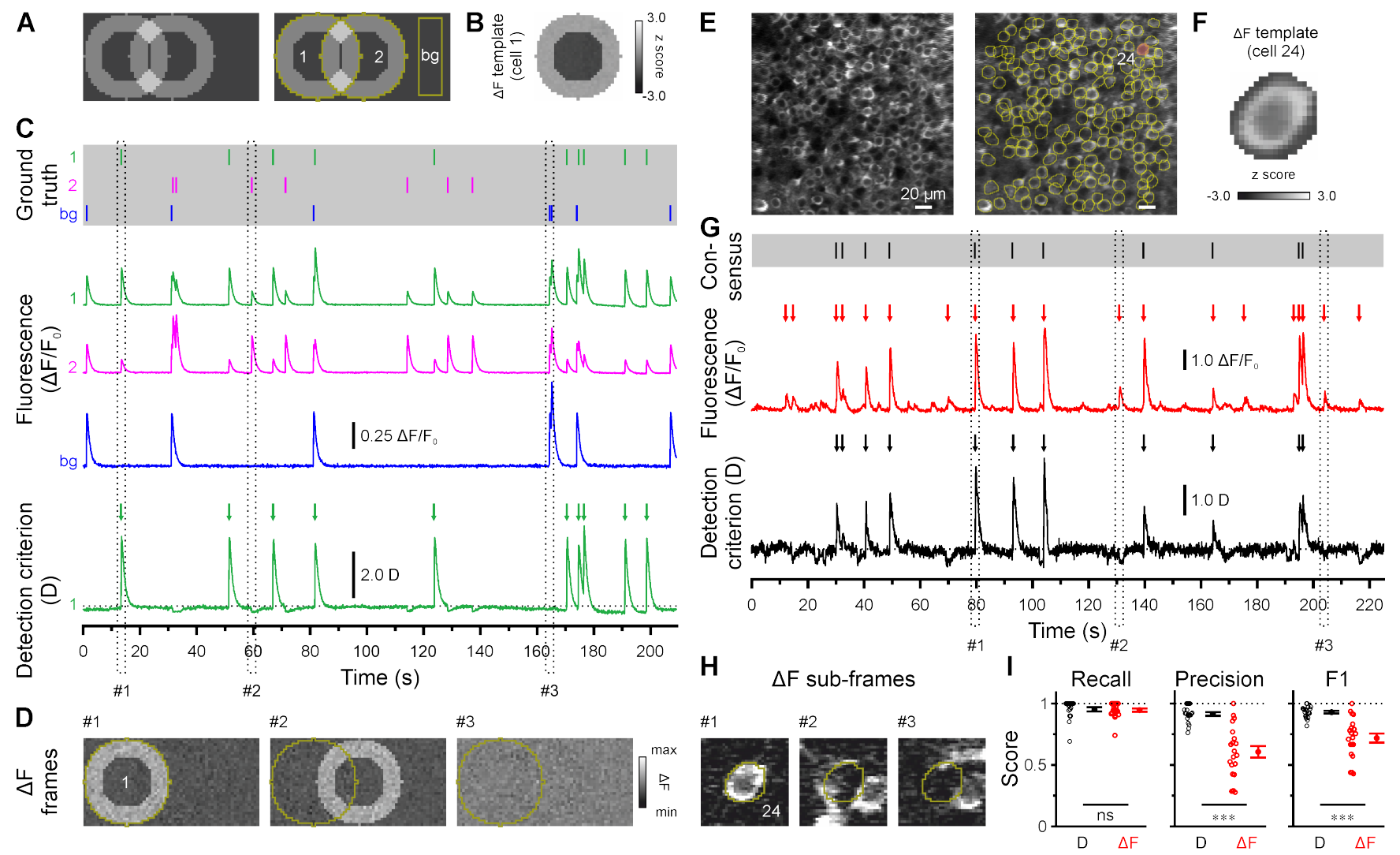

Figure 1. CATHARSiS enables reliable CaT detection in densely labeled tissue. (A) Resting image of two partially overlapping simulated cells (left) and regions of interest (ROIs) used for analysis (right). bg - background. (B) $\Delta \mathrm{F}$ template of cell 1. (C) Top, simulated trains of action potentials. Middle, relative changes from baseline fluorescence $\left(\Delta F / F_{0}\right)$ of ROIs shown in $A$. Bottom, detection criterion (D) for cell 1 and corresponding CaT onsets retrieved by CATHARSiS (arrows). (D) Sample $\Delta \mathrm{F}$ images of three individual frames at time points indicated in $\mathrm{C}$. Spikes in cell 1 or 2 translated into ring-shaped increases in $\Delta \mathrm{F}$, whereas those induced by bg spikes were applied to the entire field of view. (E) Resting GCaMP6s fluorescence image (left) and ROls used for analysis (right). (F) $\Delta \mathrm{F}$ template of the cell indicated in E. (G) Top, consensus visual annotation by two human experts for the same cell. Middle, $\Delta \mathrm{F} / \mathrm{F}_{0}$ and detected event onsets (red arrows). Bottom, detection criterion (D) and 
745 corresponding CaT onsets retrieved by CATHARSiS (black arrows). (H) Sample $\Delta \mathrm{F}$ images of

746 three individual frames at time points indicated in G. Note that frames \#2 and \#3 led to false

747 positive results if event detection was performed on mean $\Delta \mathrm{F}$, but not if performed on $\mathrm{D}$. (I)

748 Quantification of recall, precision and F1 score for event detection based on D (i.e.

749 CATHARSiS) and mean $\Delta \mathrm{F}$, respectively. Each open circle represents a single cell. Data are

750 presented as mean \pm SEM. ns - not significant. ${ }^{* * *} \mathrm{P}<0.001$. See also Table S1. 

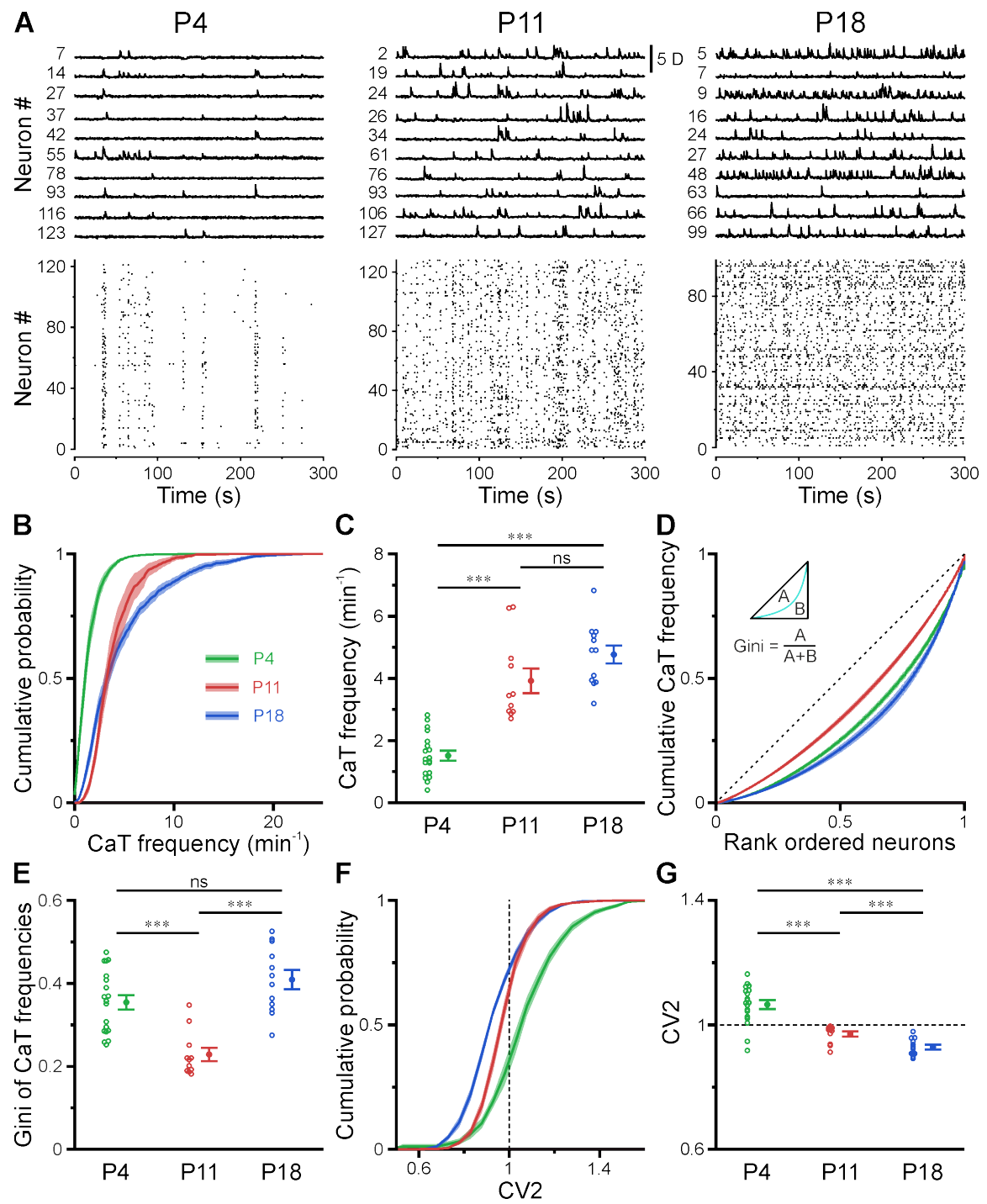

Figure 2. A transient period of firing equalization during CA1 development in vivo. (A) Sample $D(t)$ traces (top) and raster plots showing reconstructed CaT onsets (bottom). Note that cellular firing undergoes a developmental transition from synchronized-discontinuous to desynchronized-continuous activity. (B) Cumulative probability of CaT frequencies. (C) Mean CaT frequencies per FOV. (D) Lorenz curves of CaT frequencies. Line of equality (dotted) represents the case that all neurons have equal CaT frequencies. Inset depicts Gini coefficient calculation. (E) Mean Gini coefficients per FOV. (F) Cumulative probability of mean CV2 of inter-CaT intervals. Note that, at P11, CV2 distribution is narrower and centered around 1. (G) Mean CV2 per FOV. For a Poisson process, CV2 = 1 (dotted line). Each open 
bioRxiv preprint doi: https://doi.org/10.1101/2021.05.28.446133; this version posted May 29, 2021. The copyright holder for this preprint (which was not certified by peer review) is the author/funder. All rights reserved. No reuse allowed without permission.

761 circle represents a single FOV. Data are presented as mean \pm SEM. ns - not significant. P4:

762 P3-4, P11: P10-12, P18: P17-19, ${ }^{* * *} \mathrm{P}<0.001$. See also Table S2. 

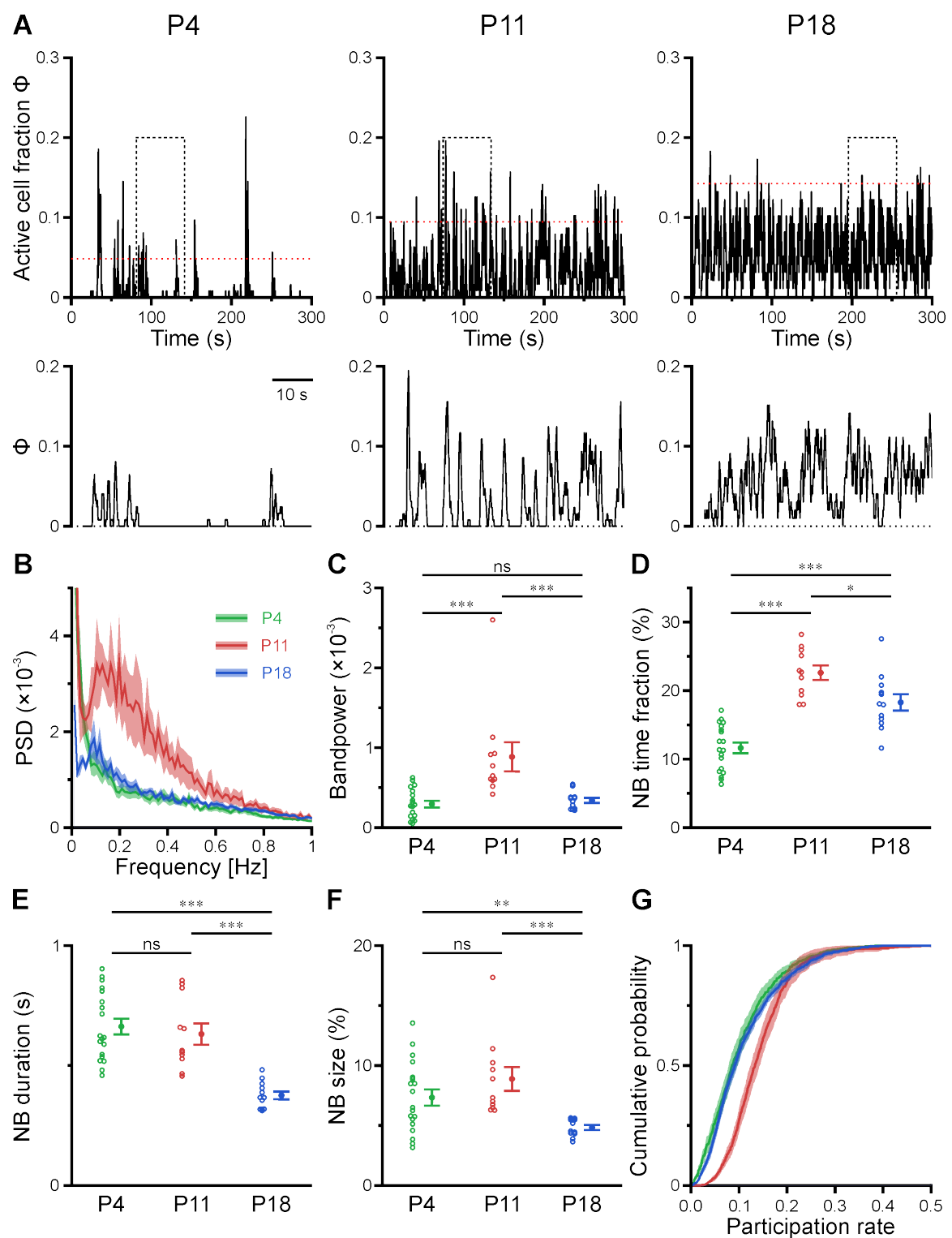

Figure 3. CA1 undergoes a transient enhanced bursting period in vivo. (A) Sample traces of the fraction of active cells $\Phi(\mathrm{t})$. Bottom traces show time periods marked on top (dotted rectangle) at higher temporal resolution. The red dotted line indicates the activity-dependent threshold for NB detection. (B) Power spectral density of $\Phi(\mathrm{t})$. (C) Bandpower of $\Phi(\mathrm{t})$ in the $0.1-0.5 \mathrm{~Hz}$ range. (D) The fraction of time that the network spent in NBs peaked at P11. (E) The average NB duration is lowest at P18. (F) Quantification of NB size as the mean fraction of active neurons (corrected for burst threshold as indicated in A). (G) Cumulative probability of the fraction of NBs that each cell is participating in. Each open circle represents a single 
bioRxiv preprint doi: https://doi.org/10.1101/2021.05.28.446133; this version posted May 29, 2021. The copyright holder for this preprint (which was not certified by peer review) is the author/funder. All rights reserved. No reuse allowed without permission.

772 FOV. Data are presented as mean \pm SEM. ns - not significant. P4: P3-4, P11: P10-12, P18:

$773 \mathrm{P} 17-19,{ }^{* * *} \mathrm{P}<0.001 .{ }^{* *} \mathrm{P}<0.01 .{ }^{*} \mathrm{P}<0.05$. See also Table S3. 

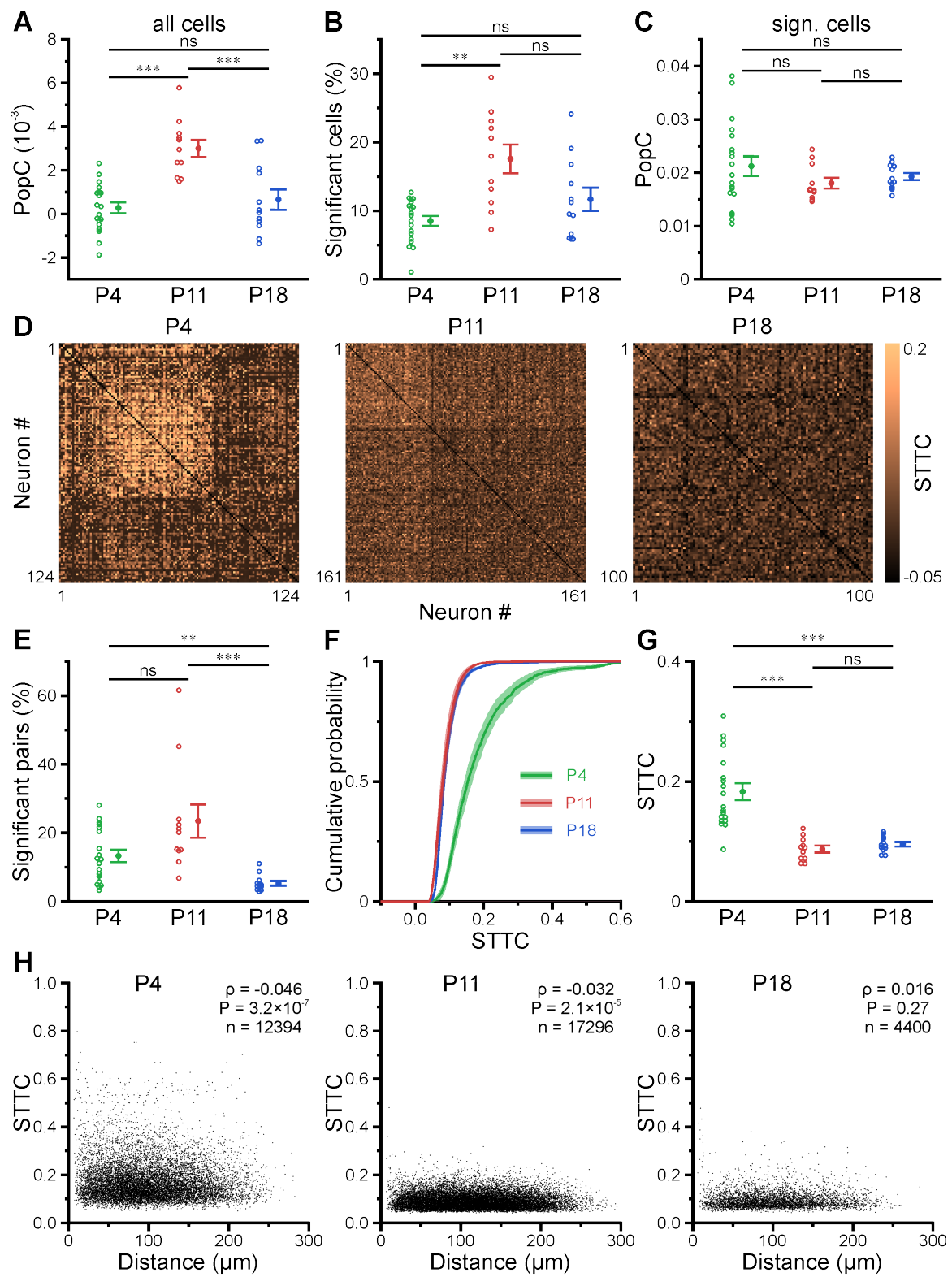

Figure 4. Enhanced population coupling underlies network burstiness in the second postnatal week in vivo. (A) The mean population coupling (PopC) index peaked at P11. (B) Mean fraction of cells with significant PopC. (C) Mean PopC index of significantly coupled cells only. (D) Sample STTC matrices (re-ordered). (E) Mean fraction of cell pairs having a significant STTC. (F) Cumulative probability of STTCs of significantly correlated cell pairs only. (G) Mean STTCs of significantly correlated cell pairs. (H) Relationship between STTC and Euclidean somatic distance for significantly correlated cell pairs. $\rho$ denotes the Spearman's rank 
782 correlation coefficient for all cell pairs analyzed (n) at a given age. Each open circle 783 represents a single FOV. Data are presented as mean \pm SEM. ns - not significant. P4: P3-4,

784 P11: P10-12, P18: P17-19, ${ }^{* * *} \mathrm{P}<0.001 .{ }^{* *} \mathrm{P}<0.01$. See also Table S4. 

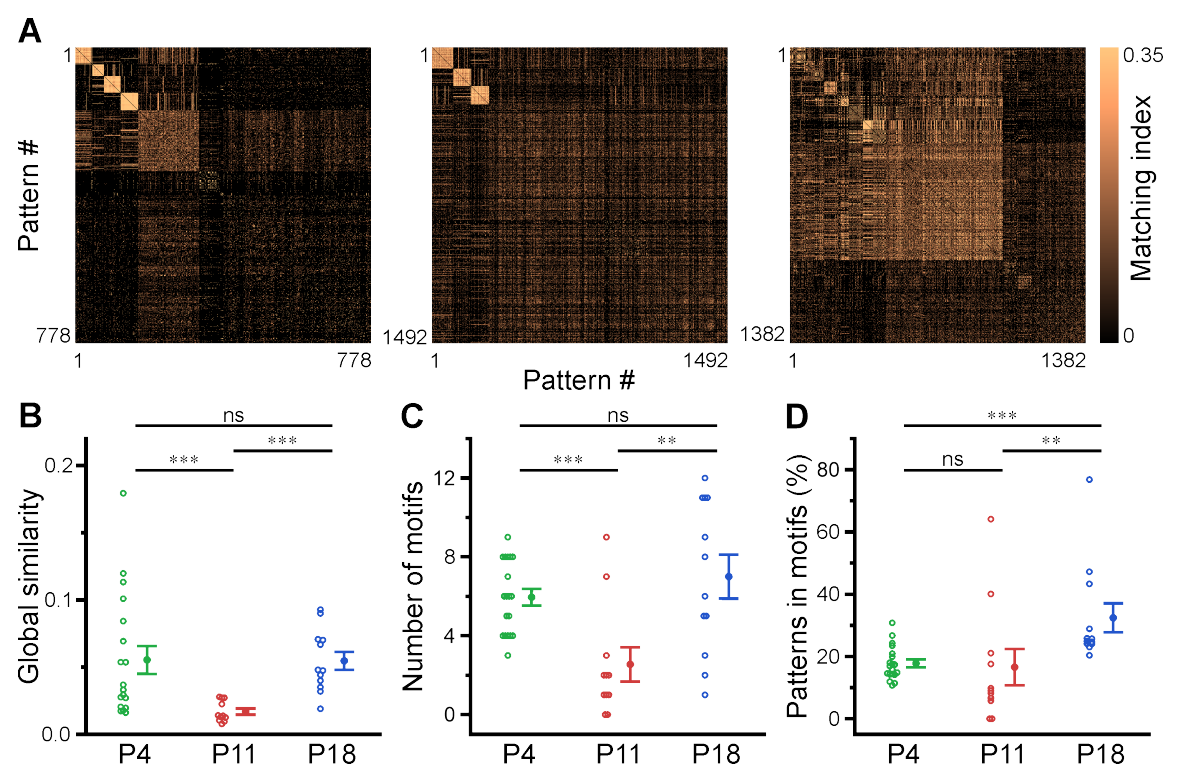

Figure 5. Motifs of CA1 network activity undergo distinct developmental alterations. (A) Similarity matrices (matching index) of binary activity patterns (re-ordered for illustration of motif detection). (B) Global similarity of activity patterns is lowest at P11. (C) The absolute number of detected motifs per FOV is lowest at P11. (D) Motif repetition quantified as the fraction of activity patterns belonging to each motif. Each open circle represents a single FOV. Data are presented as mean \pm SEM. ns - not significant. P4: P3-4, P11: P10-12, P18: P1719, ${ }^{* * *} \mathrm{P}<0.001 .{ }^{* *} \mathrm{P}<0.01$. See also Table S5. 

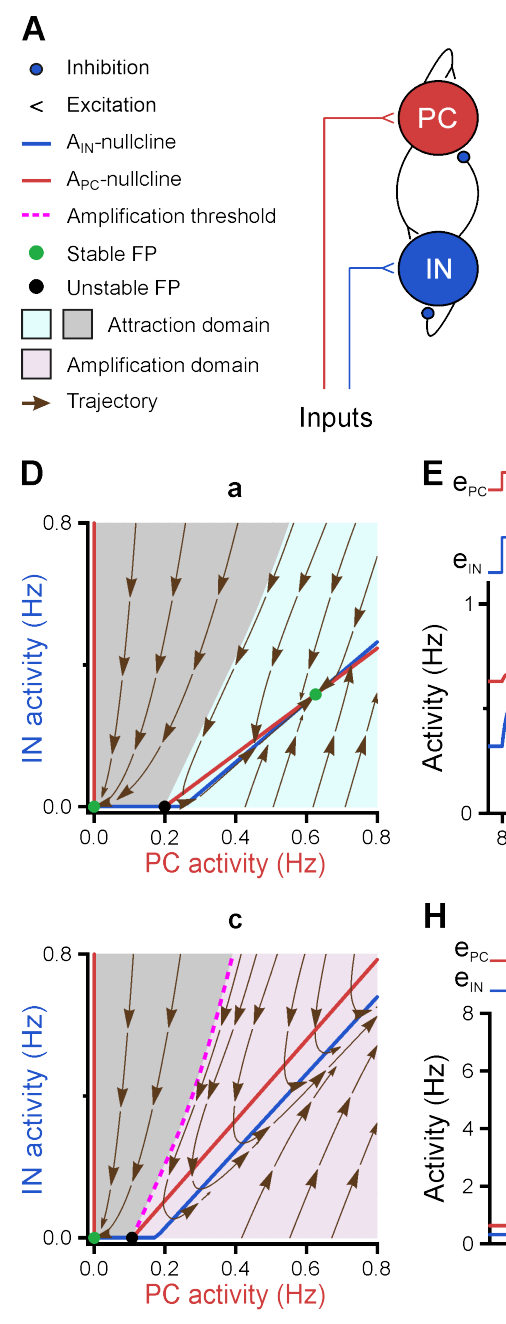
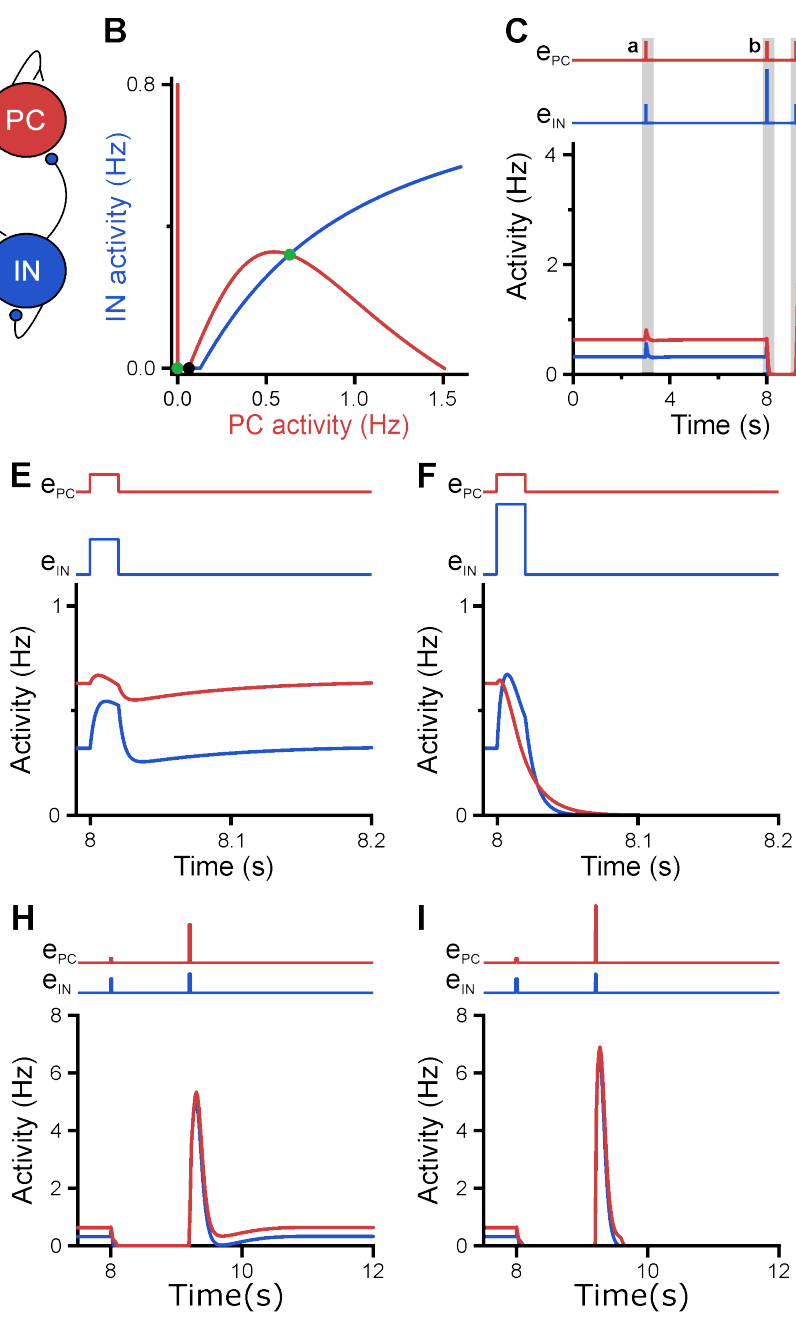
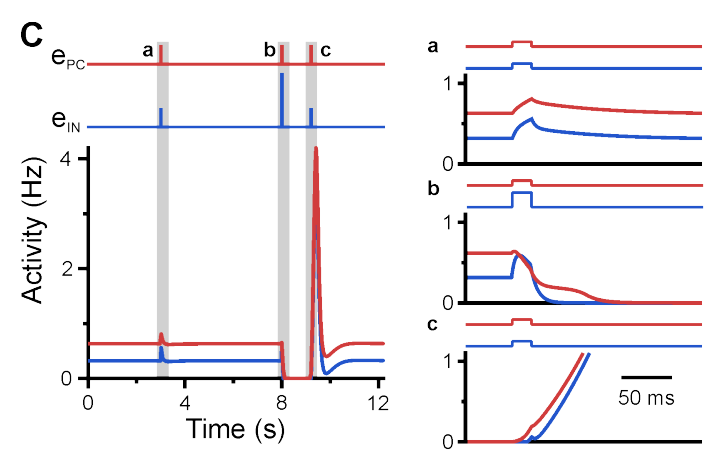

G
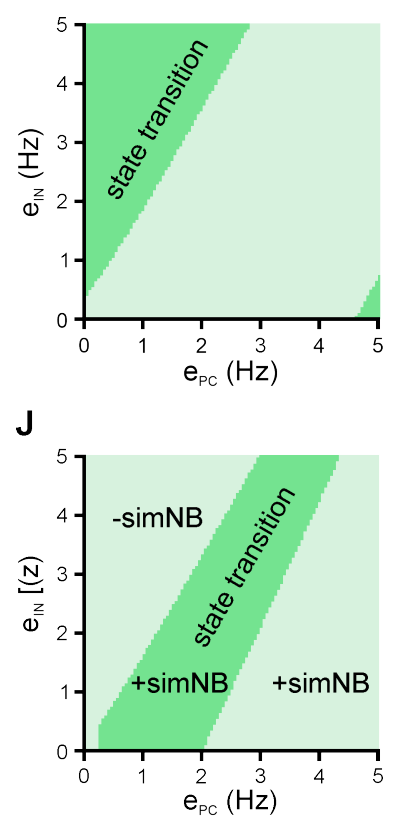

Figure 6. A neural network model with inhibitory GABA identifies intrinsic instability dynamics as key to the emergence of network bursts. (A) Schematic diagram of the STP-RNN model. (B) The $A_{1}-A_{\mathrm{p}}$-plane of the full STP-RNN's stationary dynamics. Note the presence of two stable fixed points (FPs; green dots) at silent and active states as well as the unstable FP (black dot). (C) simNB generation requires network silencing. The model was stimulated by pulse-like input to both PC and IN populations for a duration of $0.020 \mathrm{~s}$ (at $\mathrm{t}=3$ and $9.2 \mathrm{~s}$ : $e_{\mathrm{P}}=e_{\mathrm{I}}=0.25 ;$ at $\left.\mathrm{t}=8 \mathrm{~s}: e_{\mathrm{P}}=0.25, e_{\mathrm{I}}=0.75\right)$. Zoom-in of the activity around the stimulation times at active ( $a$ and $b$ ) and silent (c) states are shown in right panels. Input time series are shown on top of the plots. (D) The presence of an amplification domain in the initial phase of 
network firing dynamics enables the emergence of simNBs. The $A_{1}-A_{\mathrm{p}}$-plane of the STP-RNN with synaptic efficacies frozen at active ( $a$, top) and silent (b, bottom) states, right before input arrival. (E-G) Transition from active to silent state requires specific input ratios. Input delivered at $\mathrm{t}=8 \mathrm{~s}$. (E) A failed transition: $e_{\mathrm{P}}=0.25, e_{\mathrm{I}}=0.5$. (F) A successful transition: $e_{\mathrm{P}}=0.25, e_{\mathrm{I}}=1$. (G) A color-coded matrix of successful (dark green) and failed (light green) transitions to the silent state in response to different combinations of $e_{\mathrm{p}}$ and $e_{\mathrm{I}}$ amplitudes. $(\mathrm{H}-\mathrm{J})$ Both the transition from the silent to the active state and the simNB generation require specific input ratios. Input delivered at $t=9.2 \mathrm{~s}$. Same as $E-G$, but for the backward transition to the active state. + simNB and -simNB indicate the emergence and absence of bursts. 

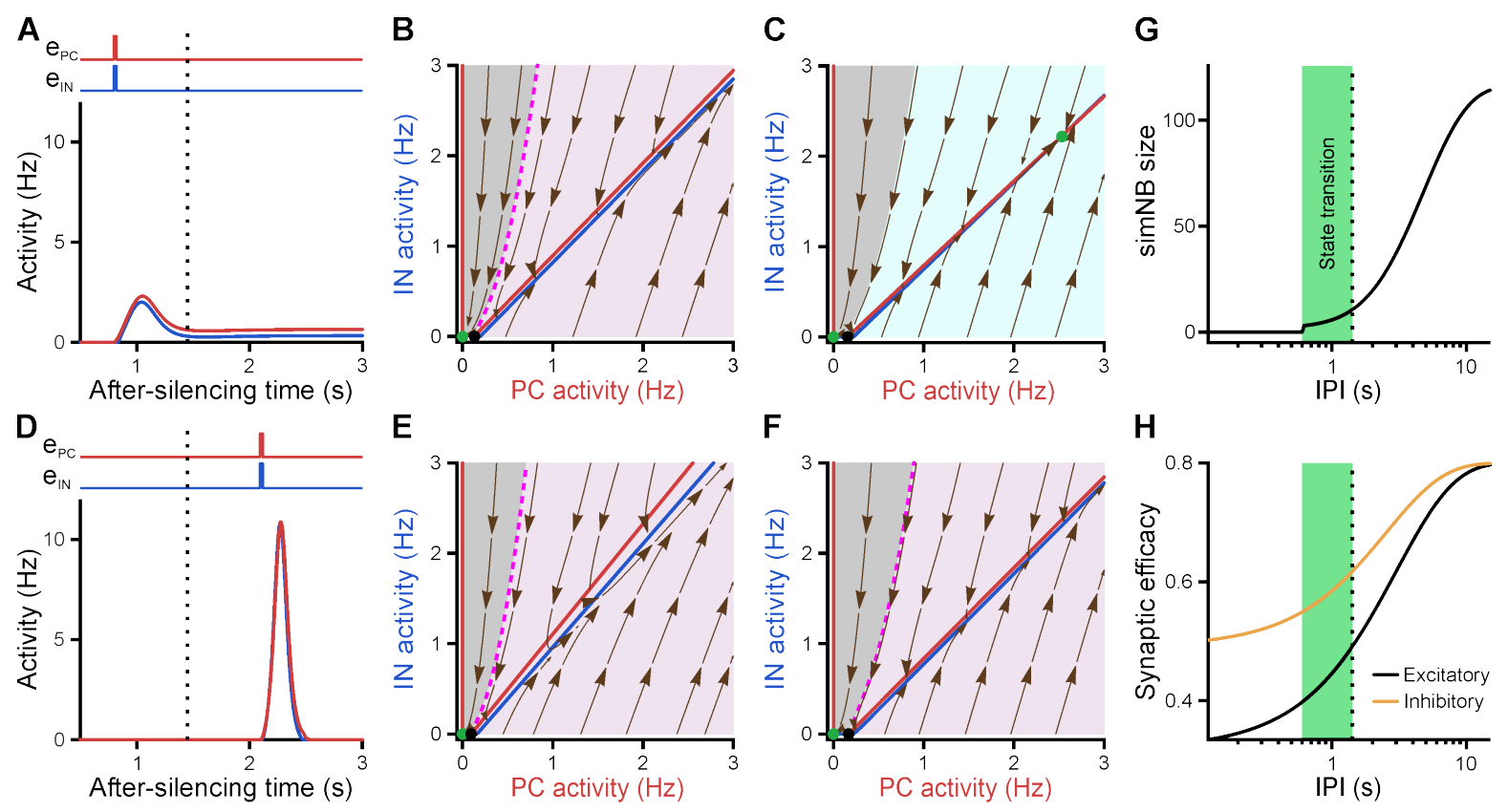

Figure 7. Internal deadline of state transitions. (A-C) Input delivered to the network before the deadline can move it to active state. (A) A successful transition. The input delivered at $t=$ $0.8 \mathrm{~s} ; e_{\mathrm{p}}=0.25, e_{\mathrm{I}}=0.25$. (B) The $A_{\mathrm{r}}-A_{\mathrm{p}}$-plane of the STP-RNN with synaptic efficacies frozen at the silent state right before the input arrival. (C) Same as B, but frozen at the peak of the network burst (i.e. simNB) shown in A. Note the presence of the transient stable FP (nonorigin green dot), which triggers the transitioning to the active state. (D-F) Once the deadline is missed, the network cannot be moved to the active state by the subsequent input. Same as $\mathrm{A}-\mathrm{C}$, but the input delivered at $\mathrm{t}=2.1 \mathrm{~s}$. Note the absence of a non-origin transient stable FP in $F$, in contrast to $C$. (G) The simNB size and network transition to the active state depend on the inter-pulse intervals (IPI: the arrival time of the next input relative to the silencing time of the network). simNB size is computed as the maximum of $A_{1}+A_{\mathrm{p}}$ after the secondary input. Note the presence of a short window for transitioning to the active state. $e_{\mathrm{P}}=0.25, e_{\mathrm{I}}=0.25$. (H) Same as G, but for the non-scaled efficacies of GABAergic $\left(u_{I} X_{I}\right.$; orange; see Methods) and glutamatergic $\left(u_{p} x_{p}\right.$; black) synapses, right before the arrival of the secondary input. (A, 
bioRxiv preprint doi: https://doi.org/10.1101/2021.05.28.446133; this version posted May 29, 2021. The copyright holder for this preprint (which was not certified by peer review) is the author/funder. All rights reserved. No reuse allowed without permission.

$827 \mathrm{D}, \mathrm{G}, \mathrm{H})$ The dotted line at $\mathrm{t}=1.45 \mathrm{~s}$ depicts the internal deadline. 


\section{Methods}

\section{Animals}

All animal procedures were performed with approval of the local government (Thüringer Landesamt für Verbraucherschutz, Bad Langensalza, Germany) and complied with European Union norms (Directive 2010/63/EU). Animals were housed in standard cages with 14h/10h light/dark cycles. Emx1 $1^{\text {REScre }}$ (stock no. 005628) and GCaMP6s ${ }^{L S L}$ (Ai96, stock no. 024106) mice were originally obtained from The Jackson Laboratory. Double heterozygous offspring $\left(E m \times 1^{I^{R E S C r e / w t}}: G C a M P 6 s^{L S L / w t}\right)$ was used for experiments at P3-4 ('P4'), P10-12 ('P11') and P17-19 ('P18'). Mice of either sex were used.

\section{Surgical preparation, anesthesia and animal monitoring for in vivo imaging}

30 minutes before starting the preparation, $200 \mathrm{mg} / \mathrm{kg}$ metamizol (Novacen) was subcutaneously injected for analgesia. Animals were then placed onto a warm platform and anesthetized with isoflurane (3.5\% for induction, $1-2 \%$ for maintenance) in pure oxygen (flow rate: $1 \mathrm{l} / \mathrm{min}$ ). The skin overlying the skull was disinfected and locally infiltrated with $2 \%$ lidocaine (s.c.) for local analgesia. Eyes of P17-19 were lubricated with a drop of eye ointment (Vitamycin). Scalp and periosteum were removed, and a custom-made plastic chamber with a central borehole $(\varnothing 2.5-4 \mathrm{~mm})$ was fixed on the skull using cyanoacrylate glue (Uhu) (P4: $3.5 \mathrm{~mm}$ rostral from lambda and $1.5 \mathrm{~mm}$ lateral from midline; P11: $3.5 \mathrm{~mm}$ rostral from lambda and $2 \mathrm{~mm}$ lateral from midline; P18: $3.5 \mathrm{~mm}$ rostral from lambda and 2.5 $\mathrm{mm}$ lateral from midline).

For the hippocampal window preparation (Mizrahi et al., 2004), the plastic chamber was tightly connected to a preparation stage and subsequently perfused with warm artificial 
cerebrospinal fluid (ACSF) containing (in mM): $125 \mathrm{NaCl}, 4 \mathrm{KCl}, 25 \mathrm{NaHCO}_{3}, 1.25 \mathrm{NaH}_{2} \mathrm{PO}_{4}$,

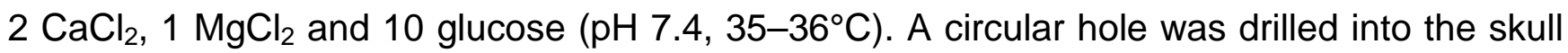
using a tissue punch (outer diameter $1.8 \mathrm{~mm}$ for P4 and $2.7 \mathrm{~mm}$ for P11 and P18 mice). The underlying cortical tissue and parts of corpus callosum were carefully removed by aspiration using a vacuum supply and a blunt $27 \mathrm{G}$ or $30 \mathrm{G}$ needle. Care was taken not to damage alveus fibers. As soon as bleeding stopped, the animal was transferred to the microscope stage.

During in vivo recordings, body temperature was continuously monitored and maintained at close to physiological values $\left(36-37^{\circ} \mathrm{C}\right)$ by means of a heating pad and a temperature sensor placed below the animal. Spontaneous respiration was monitored using a differential pressure amplifier (Spirometer Pod and PowerLab 4/35, ADInstruments). Isoflurane was discontinued after completion of the surgical preparation and gradually substituted with the analgesicsedative nitrous oxide (up to the fixed final $\mathrm{N}_{2} \mathrm{O} / \mathrm{O}_{2}$ ratio of $3: 1$, flow rate: $1 \mathrm{l} / \mathrm{min}$ ). Experiments started 60 min after withdrawal of isoflurane. At the end of each experiment, the animal was decapitated under deep isoflurane anesthesia.

\section{Two photon $\mathrm{Ca}^{2+}$ imaging in vivo}

After transferring the animal to the microscope stage, ACSF was removed and the hippocampal window was filled up with a droplet of agar $(1 \%$, in $0.9 \% \mathrm{NaCl})$ and covered with a cover glass. As soon as the agar solidified, the chamber was again perfused with ACSF.

Imaging was performed using a Movable Objective Microscope (Sutter Instrument) equipped with two galvanometric scan mirrors $(6210 \mathrm{H}$, MicroMax $673 X X$ Dual Axis Servo Driver, Cambridge Technology) and a piezo focusing unit (P-725.4CD PIFOC, E-665.CR amplifier, Physik Instrumente) controlled by a custom-made software written in LabVIEW 2010 (National Instruments) (Kummer et al., 2015) and MPScope (Nguyen et al., 2006). 
Fluorescence excitation at $920 \mathrm{~nm}$ was provided by a tunable Ti:Sapphire laser (Chameleon Ultra II, Coherent) using a 20×/1.0 NA water immersion objective (XLUMPLFLN 20XW, Olympus). Emission light was separated from excitation light using a 670-nm dichroic mirror (670 DCXXR, Chroma Technology), short-pass filtered at $680 \mathrm{~nm}$ and detected by a photomultiplier tube (12 bit, H10770PA-40, Hamamatsu). Data were acquired using two synchronized data acquisition devices (NI 6110, NI 6711, National Instruments). Sampling rate was set to $11.63 \mathrm{~Hz}(256 \times 256$ pixels, $248 \times 248 \mu \mathrm{m})$. For each animal, spontaneous activity was recorded within 3-5 fields of view, each one usually for $\sim 20 \mathrm{~min}$.

\section{Quantification and statistical analysis}

Preprocessing

Image stacks were registered using NoRMCorre (Pnevmatikakis et al., 2017). For residual drift detection, a supporting metric was calculated as the Pearson correlation coefficient of the binarized template image used for stack registration and the binarized images of the registered image stack. Time periods with residual drift were then visually identified (by inspecting the supporting metric and the aligned image stack) and considered as missing values in subsequent analyses. Raw regions of interest (ROIs) were manually drawn around the somata of individual CA1 PCs using Fiji.

\section{CATHARSiS - Calcium transient detection harnessing spatial similarity}

For the detection of CaTs in densely labeled tissue, we devised CATHARSiS (alcium transient detection harnessing spatial similarity). CATHARSiS makes use of the fact that spike-induced somatic GCaMP signals $(\Delta \mathrm{F})$ are spatially non-uniform and characteristic of a given cell. CATHARSiS comprises three major steps: (1) the generation of a spatial $\Delta \mathrm{F}$ 
template representing the active cell, (2) the computation of a detection criterion $D(t)$ for each time point (frame) and (3) the extraction of CaT onsets. All analyses were performed using custom scripts in Matlab and Fiji.

Ad (1): For each ROI, we first obtained the mean $F(t)$ by frame-wise averaging across all pixels of that ROI. We then computed the first derivative of $F(t)$ and smoothed it using a second order Savitzky-Golay algorithm (window length, 6 frames), thus yielding $\dot{F}(t)$. We then determined eight candidate CaT onsets by extracting the frame numbers corresponding to the eight $\dot{F}(\mathrm{t})$ peaks having the largest amplitude. This step was performed in an iterativedescending manner by starting with the largest $F(t)$ peak. For each peak, we defined a minimum time difference (5 frames) to all subsequently extracted peaks, so as to avoid extracting nearby frames belonging to the same CaT. For each candidate CaT onset, we then computed the corresponding spatial $\Delta F$ (average of five successive frames). To this end, we first radially expanded the raw ROI by two pixels using the Euclidian distance transform (we found that this increased detection reliability due to enhanced spatial contrast). Resting fluorescence $F_{0}(t)$ was defined as the moving median over 500 frames. Eight candidate $\Delta F$ templates were obtained by converting raw $\Delta \mathrm{F}$ values into z-scores. Based on visual inspection, we next rejected those candidate $\Delta \mathrm{F}$ templates that putatively reflected activation of optically overlapping somata and/or neurites. If all candidate $\Delta \mathrm{F}$ templates had been rejected, the cell was excluded from further analysis; otherwise, the remaining candidate $\Delta \mathrm{F}$ templates were averaged to obtain the final $\Delta \mathrm{F}$ template representing the active cell.

Ad (2): For each ROI (spatially expanded as above), we extracted its spatial $\Delta \mathrm{F}$ for all frames in the image stack. Next, the spatial $\Delta \mathrm{F}$ template representing the active cell was optimally scaled to fit its $\Delta \mathrm{F}$ in each recorded frame. Based on the optimum scaling factor and the goodness of the fit, a detection criterion $D(t)$ was computed for each time point. Here, $D(t)$ 
was defined without modification as previously described for the temporal domain (Clements et al., 1997).

Ad (3): For each ROI, CaT onsets were extracted from $D(t)$ using UFARSA, a generalpurpose event detection routine (Rahmati et al., 2018). To this end, we slightly modified the original UFARSA approach in two ways. I) Following the smoothing step implemented in UFARSA, all negative values were set to zero, as we found in our perliminary analysis that negative-to-positive transitions occasionally resulted in false positive events. II) We introduced a lower bound for the leading threshold, so as to minimze potential false positive events. Reconstructed CaT onsets were translated into a binary activity vector and used for the following analyses.

\section{Firing irregularity}

For each cell, we quantified the irregularity of its CaT onsets (i.e. firing times) using CV2, as a local and relatively rate-independent measure of spike time irregularity (Holt et al., 1996; Ponce-Alvarez et al., 2010): CV2 $=\frac{1}{K-1} \sum_{k=1}^{K-1} \frac{2\left|I C I_{k+1}-I C I_{k}\right|}{I C I_{k+1}+I C I_{k}}$, where $I C I_{k}$ and $I C I_{k+1}$ are the $k$ th and $(k+1)$ th inter-CaT intervals of the cell, and $K$ is the total number of its ICI s. To achieve more robust results cells with less than ten ICI s were excluded from this analysis.

\section{Network bursts}

Network bursts (NBs) were defined as a significant co-activation of cells as follows: (1) To account for some temporal jitter in the detection of CaT onsets, we calculated the moving maximum of the binary activity vectors of all cells in a given FOV over a sliding window of three frames. We then computed the mean across the resulting activity vectors of all individual cells to obtain the empirical fraction of active cells per frame $\Phi(t)$. (2) We randomly 
shuffled CaT onsets of all cells (uniform distribution; 1,000 times), computed the surrogate $\Phi(t)$ (as above) and defined the 99.99th percentile of all surrogate $\Phi(t)$ as the threshold for NB detection. The NB threshold was determined separately for each FOV, so as to account for different mean CaT frequencies. (3) Any frame with an empirical $\Phi(t)$ exceeding the threshold was considered as belonging to an NB. In the resulting binary NB vectors, 0-1 transitions were defined as NB onsets and 1-0 transitions as NB offsets. Using the binary NB vectors, we extracted (i) the relative time the network spent in NBs and (ii) the average NB duration. NB size was defined as the fraction of cells which were active in at least one frame of a given NB, corrected for the chance level of co-activation by subtracting the NB threshold.

\section{Power analysis}

To account for missing values representing the residual drift periods (see above), spectral power of the fraction of active cells $\Phi(\mathrm{t})$ was estimated by computing the Lomb-Scargle periodogram (Matlab, MathWorks).

\section{Pairwise correlations}

Spike-time tiling coefficients (STTCs) were computed for all possible cell pairs with a synchronicity window of three frames ( 258 ms) using custom written code (Matlab, MathWorks) (Cutts et al., 2014). STTCs derived from measured data were compared to those from surrogate data obtained by randomly shuffling (uniform distribution; 1,000 times) CaT onsets of all cells, separately. This randomization kept the mean CaT frequency of each cell unchanged.

\section{Population coupling}


To quantify the degree of coupling of each cell to the overall population firing activity we computed its population coupling, PopC (Okun et al., 2015; Sweeney et al., 2020). To this end, for each cell, we first smoothed its binary vector (see above) and the summed vector of the rest of population, followed by computing PopC as the Pearson correlation coefficient between these two vectors. For smoothing, we used a Gaussian kernel with $S D=3$ frames. To assess the significance of the PopCs (i.e. being beyond chance) we generated surrogate data by binning the raster matrix along time-axis; non-overlapping bins with a size of 10 frames (ca. $\sqrt{12} S D$, according to (Kruskal et al., 2007)). We randomly exchanged CaT onsets across active cells within each bin (500 times), thereby effectively preserving the CaT frequency of each cell as well as the local summed activity of the population. For each cell, using its surrogates, we determined the significance of its empirical PopC (95 ${ }^{\text {th }}$ percentile). Moreover, when reporting the PopC of each cell, we subtracted the mean of its surrogate PopCs, in order to account for the potential differences in population activity levels of different FOVs (for a similar approach see (Okun et al., 2015; Sweeney et al., 2020)). Cells with less than five CaTs were excluded from this analysis, to increase robustness of our results.

\section{Motifs of population activity}

To identify the specific cellular activation patterns recurring over time (i.e. motifs of population activity) we used an eigendecomposition-based clustering method (Li et al., 2010; Patel et al., 2015). To this end, we first divided the recording time into non-overlapping windows with a size of 10 frames, and assigned 1 and 0 to cells which were active or silent during each bin. This converts the raster matrix to a sequence of binary vectors (i.e. spatial patterns), where each pattern has a size of $\mathrm{Nx1}$ ( $\mathrm{N}$ is the number of analyzed cells in the FOV). We then computed the degree of similarity between all possible pairs of these patterns using matching 
index (Romano et al., 2015): $\mathrm{MI}_{i j}=2 \frac{\left|P a t_{i} \cap P a t_{j}\right|}{\left|P a t_{i}\right|+\left|P a t_{j}\right|}$, where Pat $_{i}$ and Pat $_{j}$ are the ith and jth binary cellular activation patterns (vectors), and the norms are equal to the number of ones (i.e. active cells) in each vector. MI ranges from 0 (no similarity) to 1 (perfect similarity), and in particular approximates the number of common neuronal activations (i.e. common ones) between pattern pairs; for more details see (Romano et al., 2015; Sporns et al., 2007). Accordingly, for each FOV, we obtained a similarity matrix of size $\mathrm{P} \times \mathrm{P}$, where $\mathrm{P}$ indicates the number of patterns. The rows and columns relating to the silent-pattern pairs were excluded, as they were giving rise to an undefined value (i.e. 0 divided by 0 ). We used the $\mathrm{MI}$ matrix as the input to the eigendecomposition clustering method. Briefly, this method decomposes a given similarity matrix (here, MI matrix) into a set of eigenvalues and eigenvectors: The number of significantly large eigenvalues determines the number of motifs, and their corresponding eigenvectors contain the information about motif structure (i.e. the set of patterns belonging to each motif). The largest eigenvalue is proportional to the global similarity among all patterns. As the surrogate data for testing the statistical significance of the eigenvalues and also computing a normalized unbiased value of global similarity index, we used the randomly shuffled CaT onsets (see above), based on which we repeated the binning and computation of MI matrices (500 times). This procedure enabled us to identify the motifs of cellular activation patterns, which occurred beyond chance level. For more details about the clustering method and its mathematical description see (Li et al., 2010).

\section{Computational modeling of a developing neural network with inhibitory GABA}

Overview. To gain insights into the mechanisms and functional role of the observed network burstiness during the emergence of synaptic inhibition in CA1, we used computational 
modeling and stability analysis. For this purpose, we employed a recently established model of a recurrent neural network (RNN) for first postnatal month development (Rahmati et al., 2017). It is an extended Wilson-Cowan-type model (Tsodyks et al., 1998), and benefits from being biophysically interpretable and mathematically accessible. Recently, this model was also adapted successfully to explain key dynamics and mechanisms of GDPs in neonatal CA1 with excitatory GABA signaling during the first postnatal week (Flossmann et al., 2019). However, in accordance with previous reports and our present experimental data for the second postnatal week, we here use the model with mainly two specific cellular properties: I) GABAergic synapses are considered inhibitory (Kirmse et al., 2015; Valeeva et al., 2016; Murata et al., 2020), and II) the mean spontaneous firing activity of PCs is effectively non-zero (Fig. 2C). In the following, after providing the mathematical description of the model, we describe the mathematical components used for its stability analysis. For more details about the model and the approach see (Rahmati et al., 2017).

Model description. The model is a mean-field network model of mean firing activity rates of two spatially localized, homogeneous glutamatergic and GABAergic cells (here, pyramidal (PC) and interneuron (IN) populations) that are recurrently connected (Fig. 6A). The model incorporates two short-term synaptic plasticity (STP) mechanisms, namely short-term synaptic depression (STD) and facilitation (STF), which render the synaptic efficacies dynamic over time. Hence, we call the network hereafter STP-RNN. The equations governing the mean-field dynamics of the STP-RNN (10D) are (dots denote the time derivatives and, hereafter, PC and IN are abbreviated as P and I for readability) (Rahmati et al., 2017):

$$
\begin{aligned}
& \tau_{\mathrm{P}} \dot{A}_{\mathrm{P}}(t)=-A_{\mathrm{P}}(t)+f_{\mathrm{P}}\left(J_{\mathrm{PP}} u_{\mathrm{PP}}(t) x_{\mathrm{PP}}(t) A_{\mathrm{P}}(t)-J_{\mathrm{PI}} u_{\mathrm{PI}}(t) x_{\mathrm{PI}}(t) A_{\mathrm{I}}(t)+e_{\mathrm{P}}(t)\right)=-A_{\mathrm{P}}(t)+f_{\mathrm{P}}\left(h_{\mathrm{P}}\right) \\
& \tau_{\mathrm{I}} \dot{A}_{\mathrm{I}}(t)=-A_{\mathrm{I}}(t)+f_{\mathrm{I}}\left(J_{\mathrm{IP}} u_{\mathrm{IP}}(t) x_{\mathrm{IP}}(t) A_{\mathrm{P}}(t)-J_{\mathrm{II}} u_{\mathrm{II}}(t) x_{\mathrm{II}}(t) A_{\mathrm{I}}(t)+e_{\mathrm{I}}(t)\right)=-A_{\mathrm{I}}(t)+f_{\mathrm{I}}\left(h_{\mathrm{I}}\right) \\
& \dot{x}_{\mathrm{ij}}=\tau_{\mathrm{rij}}^{-1}\left(1-x_{\mathrm{ij}}(t)\right)-u_{\mathrm{ij}}(t) x_{\mathrm{ij}}(t) A_{\mathrm{j}}(t) \\
& \dot{u}_{\mathrm{ij}}=\tau_{\mathrm{f}_{\mathrm{ij}}}^{-1}\left(U_{\mathrm{ij}}-u_{\mathrm{ij}}(t)\right)+U_{\mathrm{ij}}\left(1-u_{\mathrm{ij}}(t)\right) A_{\mathrm{j}}(t)
\end{aligned}
$$


where $i$ and $j \in\{P, I\}$, and $j$ is the index of the presynaptic population, $A_{\mathrm{P}}$ and $A_{\mathrm{I}}$ are the average activity rates (in $\mathrm{Hz}$ ) of $\mathrm{PC}$ and $\mathrm{IN}$ populations which can be properly scaled to represent locally the average recorded activities in these populations, $x_{\mathrm{ij}}$ and $u_{\mathrm{ij}}$ are the average dynamic variables of STD and STF mechanisms, $\tau_{\mathrm{P}}$ and $\tau_{\mathrm{I}}$ are approximations to the decay time constants of the glutamatergic and GABAergic postsynaptic potentials, $\tau_{\mathrm{r}_{\mathrm{ij}}}$ is the synaptic recovery time constant of depression, $\tau_{\mathrm{f}_{\mathrm{ij}}}$ is the synaptic facilitation time constant, $U_{\mathrm{ij}}$ is analogous to the synaptic release probability, $J_{\mathrm{ij}}$ is the average maximum absolute synaptic efficacy of recurrent $(\mathrm{i}=\mathrm{j})$ or feedback $(\mathrm{i} \neq \mathrm{j})$ connections, and $e_{\mathrm{P}}$ and $e_{\mathrm{I}}$ are the external inputs received by the PC and IN populations from other brain regions or stimulation. In this work, we set the inputs to zero (for spontaneous baseline activity), or model them as excitatory pulse (with variable positive amplitude) with a duration of 20 ms thereby emulating e.g. the SPW-driven inputs to the PC and IN populations (Karlsson et al., 2006). The transformation from the summed input to each population, $h_{\mathrm{i}}$, to an activity output (in $\mathrm{Hz}$ ) is governed by the response function, $f_{\mathrm{i}}$, defined as:

$$
f_{\mathrm{i}}\left(h_{\mathrm{i}}\right)=\left\{\begin{array}{cc}
0 & \text { for } h_{\mathrm{i}} \leq \theta_{\mathrm{i}} \\
G_{\mathrm{i}}\left(h_{\mathrm{i}}-\theta_{\mathrm{i}}\right) & \text { for } \theta_{\mathrm{i}}<h_{\mathrm{i}}
\end{array}\right.
$$

where $\theta_{\mathrm{i}}$ is the population activity threshold, and $G_{\mathrm{i}}$ is the linear input-output gain above $\theta_{\mathrm{i}}$. In this work, we parameterize the STP-RNN as a network model representing mainly a stage during the second postnatal week. To do this, we mainly followed (Rahmati et al., 2017) by setting $\tau_{\mathrm{P}}=0.015 \mathrm{~s}, \quad \tau_{\mathrm{I}}=0.0075 \mathrm{~s}, \quad J_{\mathrm{PP}}=J_{\mathrm{IP}}=J_{\mathrm{P}}=6.5, \quad J_{\mathrm{II}}=J_{\mathrm{PI}}=J_{\mathrm{I}}=3, \quad \tau_{\mathrm{r}_{\mathrm{PP}}}=\tau_{\mathrm{r}_{\mathrm{IP}}}=\tau_{\mathrm{r}_{\mathrm{p}}}=3 \mathrm{~s}$, $\tau_{\mathrm{r}_{\mathrm{II}}}=\tau_{\mathrm{r}_{\mathrm{PI}}}=\tau_{\mathrm{r}_{\mathrm{I}}}=2.5 \mathrm{~S}, \quad \tau_{\mathrm{f}_{\mathrm{PP}}}=\tau_{\mathrm{f}_{\mathrm{IP}}}=\tau_{\mathrm{f}_{\mathrm{P}}}=0.4 \mathrm{~S}, \quad \tau_{\mathrm{f}_{\mathrm{II}}}=\tau_{\mathrm{f}_{\mathrm{PI}}}=\tau_{\mathrm{f}_{\mathrm{I}}}=0.4 \mathrm{~S}, \quad U_{\mathrm{PP}}=U_{\mathrm{IP}}=U_{\mathrm{P}}=0.8$ $U_{\mathrm{II}}=U_{\mathrm{PI}}=U_{\mathrm{I}}=0.8, \theta_{\mathrm{P}}=0.22, \theta_{\mathrm{I}}=0.53, G_{\mathrm{P}}=G_{\mathrm{I}}=1$, and $e_{\mathrm{P}}=e_{\mathrm{I}}=0 \mathrm{~Hz}$ (for spontaneous 
baseline activity). According to these parameter values: I) both glutamatergic and GABAergic connections will act depressing, and II) the network will spontaneously have, in addition to a silent state, an active state where both $A_{1}$ and, in particular, $A_{\mathrm{p}}$ are effectively non-zero, and III) GABAergic transmission will be inhibitory (note the positive value of $J_{\mathrm{I}}$ ). Note that points II) and III) render the model inherently different from the neonatal STP-RNN used by (Flossmann et al., 2019).

Frozen STP-RNN. A Frozen STP-RNN is obtained by freezing the synaptic efficacies of a STP-RNN; i.e. by fixing the STP variables $x_{\mathrm{ij}}$ and $u_{\mathrm{ij}}$ at the values of interest. This will convert the STP-RNN (10D; see Eq. 1) effectively to a 2D network with constant synaptic weights. As shown in (Rahmati et al., 2017) and (Flossmann et al., 2019), the Frozen STP-RNN can provide a reliable approximation to the stability behavior of a STP-RNN at the state chosen for freezing (see below). The equations governing the dynamics of a Frozen STP-RNN are:

$$
\begin{aligned}
& \tau_{\mathrm{P}} \dot{A}_{\mathrm{P}}(t)=-A_{\mathrm{P}}(t)+f_{\mathrm{P}}\left(J_{\mathrm{PP}}^{\mathrm{frz}} A_{\mathrm{P}}(t)-J_{\mathrm{PI}}^{\mathrm{FP}} A_{\mathrm{I}}(t)+e_{\mathrm{P}}(t)\right) \\
& \tau_{\mathrm{I}} \dot{A}_{\mathrm{I}}(t)=-A_{\mathrm{I}}(t)+f_{\mathrm{I}}\left(J_{\mathrm{IP}}^{\mathrm{frz}} A_{\mathrm{P}}(t)-J_{\mathrm{II}}^{\mathrm{frz}} A_{\mathrm{I}}(t)+e_{\mathrm{I}}(t)\right)
\end{aligned}
$$

where $J_{\mathrm{ij}}^{\mathrm{frz}}=J_{\mathrm{ij}} u_{\mathrm{ij}}^{\mathrm{frz}} x_{\mathrm{ij}}^{\mathrm{frz}}$, and $u_{\mathrm{ij}}^{\mathrm{frz}}$ and $x_{\mathrm{ij}}^{\mathrm{frz}}$ are the values of $u_{\mathrm{ij}}$ and $x_{\mathrm{ij}}$ (see Eq. 1 ) at the state of interest; here, at a silent state, active state, or the time of network burst's peak (see Results). Phase plane. To visualize the stability behavior of our network model, we used the phase plane analysis based on the activity rates: $A_{\mathrm{I}}-A_{\mathrm{p}}$-plane (2D). The $A_{\mathrm{I}}-A_{\mathrm{p}}$-plane sketch includes the curves of the $A_{\mathrm{p}}$-nullcline and $A_{\mathrm{f}}$-nullcline representing sets of points for which $\dot{A}_{\mathrm{p}}(t)=0$ and $\dot{A}_{\mathrm{I}}(t)=0$. Any intersection of these nullclines is called a fixed point (FP), with the stability needed to be determined (see below). For the STP-RNN, these FPs represent the steady states of the full network, i.e. the 10D STP-RNN in Eq. 1 (see also Fig. 6B). For the Frozen STP-RNN (thus, 2D; see Eq. 3) with synaptic efficacies frozen at the state of interest (e.g. 
silent state), these FPs may include that state, and possibly some other FPs which may not exist in the STP-RNN itself (e.g. see Figs. 6D and 7C). In addition to the visualization of the FPs in the $A_{1}-A_{\mathrm{p}}$-plane, we also computed the FPs by numerically solving Eq. 1 and Eq. 3 (separately) after setting the right hand side of the equations to zero. For more details see (Rahmati et al., 2017).

Stability of FPs. To determine the stability of any FP in the STP-RNN (resp. in the Frozen STP-RNN) we applied the linear stability analysis to its 10D (resp. 2D) system of equations in Eq. 1 (resp. Eq. 3): We investigated whether all eigenvalues of the corresponding Jacobian matrix have strictly negative real parts (if so, the FP is stable), or whether at least one eigenvalue with a positive real part exists (if so, the FP is unstable).

Simulations. All simulation results in this paper have been implemented as Mathematica and Matlab (MathWorks) code. For network simulations, we set the integration time-step size to 0.0002 s. In Fig. 6C, the initial conditions of the STP-RNN variables were set to those values of the spontaneous stable FP of the network at the active state.

\section{Statistical analysis}

Statistical analyses were performed using OriginPro 2018 and Microsoft Excel 2010 using the Real Statistics Resource Pack software (Release 7.2, Charles Zaiontz). Unless otherwise stated, the statistical parameter $n$ refers to the number of FOVs (P4: 19 FOVs from six animals, P11: 11 FOVs from six animals, P18: 12 FOVs from six animals). All data are reported as mean \pm standard error of the mean (SEM), if not stated otherwise. The ShapiroWilk test was used to test for normality. Homogeneity of variances was tested with the Levene's test using the median. For multi-group comparisons, analysis of variance (ANOVA) was applied for normally distributed data or the Kruskal-Wallis test for non-normally 
distributed data. In the case of unequal group variances, Welch's correction was applied for the ANOVA. Following a significant result in the ANOVA, post-hoc pairwise comparisons were performed using the Tukey-Kramer (equal variances) or the Games-Howell (unequal variances) test. Following a significant result in the Kruskal-Wallis test, post-hoc pairwise Mann-Whitney U-tests following Holm's approach were performed. P values (two-tailed tests) $<0.05$ were considered statistically significant, except for the Shapiro-Wilk test $(P<0.01)$. Details of the statistical tests applied are provided in Tables S1-S5.

\section{Data and code availability}

All datasets and codes generated during this study are available from the corresponding author upon request. 